\title{
Contour Integration
}

\author{
or \\ what is still missing in Mathematica \\ Part 1 : Residues and Contour Integration \\ Prof. Dr. Robert Kragler \\ Weingarten University of Applied Sciences \\ kragler@hs-weingarten.de
}

\begin{abstract}
:
The intention of this first part of a sequel of articles is to present an implementation for Contour Integration which is still missing in Mathematica. There had been some early attempts to establish numerical contour integration with NIntegrate and even line integrals over parametrically defined curves. But no symbolic contour integration procedure is implemented in Mathematica yet although in the Wolfram Functions Site reference is given to many integral representations for special functions in terms of contour integrals. With the package ContourIntegration.m an attempt is made to introduce a rather general procedure ContourIntegration which covers a wide class of functions for the integrand $f(z)$ (rational polynomials, products of rational and trigonometric/hyperbolic functions, rational functions consisting of trigonometric/hyperbolic functions, some special functions).
\end{abstract}




\section{口 Initialization}

In order to execute the subsequent Mathematica code with the examples given the Mathematica package ContourIntegration.m has to be loaded first. It should be located in the same subdirectory from where the current notebook ContourIntegration_P1.nb was revoked.

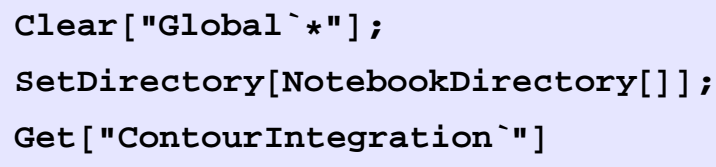

The Mathematica package comprises all definitions, procedures, replacement rules etc. which are required to run the main procedure Contour Integral etc. . After successful execution of the package the Mathematica version, date and time are shown.

VersionDateTime

Mathematica V10.4.0 for Microsoft Windows (64-bit) (February 26, 2016)

date $=$ August 14, 2016; time $=10: 50 \mathrm{~h}$

Special notations

For sake of better readability some special notations will be introduced and used throughout the notebook which are given here.

Numerical contour integrals $\oint_{\gamma} f(z) d \mathbf{z}$ and $\oint_{\mathrm{a}}^{\mathrm{b}} f(z) d \mathbf{z}$, 
Notation $\left[\oint_{\gamma_{-}} f_{-} d z_{-} \Rightarrow\right.$ NIntegrate $\left[f_{-}, \operatorname{Evaluate}\left[\operatorname{Join}\left[\left\{z_{-}\right\}, \gamma_{-}\right]\right]\right]$, WorkingForm $\left.\rightarrow t F\right]$

$\operatorname{Notation}\left[\oint_{\theta_{-}=a_{-}}^{\theta_{-}=\mathbf{b}_{-}} \mathbf{f}_{-} \mathrm{d} \mathbf{z}_{-z_{-} \rightarrow g_{-}} \Rightarrow \operatorname{NIntegrate}\left[\operatorname{Evaluate}\left[\operatorname{Simplify}\left[\frac{\mathbf{f}_{-} \operatorname{Dt}\left[\mathbf{z}_{-}\right] / . \mathbf{z}_{-} \rightarrow g_{-}}{\operatorname{Dt}\left[\theta_{-}\right]}\right]\right],\left\{\theta_{-}, \mathbf{a}_{-}, \mathbf{b}_{-}\right\}\right]\right.$, WorkingForm $\left.\rightarrow \mathrm{tF}\right]$

Line integrals $\int_{\mathcal{L}(\mathrm{t})} f(R(t)) \cdot d \mathrm{t}[R]$

Notation $\left[\int_{\mathcal{L}_{-}, \mathbf{p}_{-}} \mathbf{f}_{-} \cdot \mathrm{dt}\left[\boldsymbol{r}_{-}\right] \Leftrightarrow\right.$ LineIntegral $\left.\left[f_{-} \cdot \operatorname{Dt}\left[\mathbf{r}_{-}\right], \mathcal{L}_{-}, \mathbf{p}_{-}, \mathbf{r}_{-}\right]\right]$

Symbolic contour integrals $\oint_{\text {selPol, polRange, onoff }} f(z) d z$

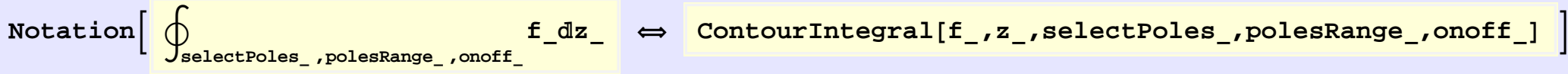

Replacement Rules

This are substitution rules for $\{\sin (\theta), \cos (\theta)\}$ and $\{\sinh (\theta), \cosh (\theta)\}$ not included in the package Contour Integration 


$$
\begin{aligned}
& z=\text { ； } \\
& \text { trigRule: }=\left\{\operatorname{Sin}\left[\theta_{-}\right] \rightarrow \frac{1}{2 \dot{i}}\left(z-\frac{1}{z}\right), \operatorname{Cos}\left[\theta_{-}\right] \rightarrow \frac{1}{2}\left(z+\frac{1}{z}\right), \operatorname{Csc}\left[\theta_{-}\right] \rightarrow \frac{2 \dot{i}}{z-\frac{1}{z}}, \operatorname{Sec}\left[\theta_{-}\right] \rightarrow \frac{2}{z+\frac{1}{z}},\right. \\
& \left.\operatorname{Tan}\left[\theta_{-}\right] \rightarrow-\dot{\mathbf{i}} \frac{\left(z-\frac{1}{z}\right)}{\left(z+\frac{1}{z}\right)}, \operatorname{Cot}\left[\theta_{-}\right] \rightarrow \dot{\mathbf{i}} \frac{\left(z+\frac{1}{z}\right)}{\left(z-\frac{1}{z}\right)}, d \theta_{-} \rightarrow \frac{1}{\dot{i} z} d z\right\} ; \quad\left(* z=e^{\dot{i} \theta} *\right) \\
& \text { hypRule: }=\left\{\sinh \left[\theta_{-}\right] \rightarrow \frac{1}{2}\left(z-\frac{1}{z}\right), \operatorname{Cosh}\left[\theta_{-}\right] \rightarrow \frac{1}{2}\left(z+\frac{1}{z}\right), \operatorname{Csch}\left[\theta_{-}\right] \rightarrow \frac{2}{\left(z-\frac{1}{z}\right)}, \operatorname{Sech}\left[\theta_{-}\right] \rightarrow \frac{2}{\left(z+\frac{1}{z}\right)}\right. \text {, } \\
& \left.\operatorname{Tanh}\left[\theta_{-}\right] \rightarrow \frac{\left(z-\frac{1}{z}\right)}{\left(z+\frac{1}{z}\right)}, \operatorname{Coth}\left[\theta_{-}\right] \rightarrow \frac{\left(z+\frac{1}{z}\right)}{\left(z-\frac{1}{z}\right)}, d \theta_{-} \rightarrow \frac{1}{z} d z\right\} ; \quad\left(* z=e^{\theta} *\right)
\end{aligned}
$$

\section{- Prolog}

The built-in Mathematica procedure NIntegrate admits the input of a (simple) contour of points in the complex plane $\mathbb{C}$ where the contour path $\gamma$ is given as a closed polygonal line. In the reference for NIntegrate one finds besides other details :

$\mathbf{N I n t e g r a t e}\left[\boldsymbol{f},\left\{\mathbf{x}_{,} \mathbf{x}_{0}, \mathbf{x}_{1}, \ldots, \mathbf{x}_{n}\right\}, \ldots\right]$ tests for singularities in a one-dimensional integral at each of the intermediate points $\boldsymbol{x}_{\boldsymbol{i}}$. If there are no singularities, the result is equivalent to an integral from $\boldsymbol{x}_{\boldsymbol{0}}$ to $\boldsymbol{x}_{\boldsymbol{n}}$. Alternatively, one can use complex numbers $z_{i}$ to specify an integration contour in the complex plane $\mathbb{C}$.

(i) Consider, for example, the contour $\gamma=\{\mathbf{1}, \boldsymbol{i}, \mathbf{- 1},-\mathbf{i}, \mathbf{1}\}$ then numical calculation of the contour integral $\frac{1}{2 \pi i} \oint_{\gamma} \frac{e^{z}}{z} d z$ yields :

$$
\frac{1}{2 \pi \dot{i}} \text { NIntegrate }\left[\frac{e^{z}}{z},\{z, 1, i,-1,-\dot{i}, 1\}\right] / / \text { ratChop }
$$


Here, Chop removes the small imaginary part resulting from numerical integration and applying Rationalize converts the approximate result from real to integer number $\mathbf{1}$. The result is agrees with the residue of the integrand :

$\operatorname{Residue}\left[\frac{e^{z}}{z},\{z, 0\}\right]$

Although the above notation using NIntegrate is quite acceptable it would be even better to use the traditional mathematical notation.

In "Tricks of the Trade" P. Abbott [1,2] and T. Bahder [3] have already shown how line integrals such as $\int_{\gamma} f(z) d z$ along the contour path $\gamma$ can be rewritten as $\oint_{\gamma_{-}} \mathbf{f}_{-} \mathbf{d} \mathbf{z} \mathbf{z}_{-}$which follows more the traditional notation of contour integrals. Using the Notation package it is straightforward defining an interpretation rule which exploits the standard syntax for numerical contour integrals

Notation $\left[\oint_{\gamma_{-}} f_{-} d z_{-} \Rightarrow\right.$ NIntegrate $\left[f_{-}\right.$, Evaluate $\left[\right.$Join $\left.\left.\left[\left\{z_{-}\right\}, \gamma_{-}\right]\right]\right]$, WorkingForm $\rightarrow$ TraditionalForm $]$

Thus, using this notation the integral can be written in a more elegant way where, in general, $\gamma$ could be any polygonal contour.

Here a diamond-like closed contour around the origin $\mathbf{( 0 , 0 )}$ is specified by points in the complex plane $\mathbb{C}$. With showPolygonalContour $\mathbf{1}$ (using ListPlot) the contour path $\gamma$ is visualized by computing the real and imaginary parts of the vertex points which define the contour. Hence, the (polygonal) contour $\gamma$ is specified and arrows show the direction of the contour path 


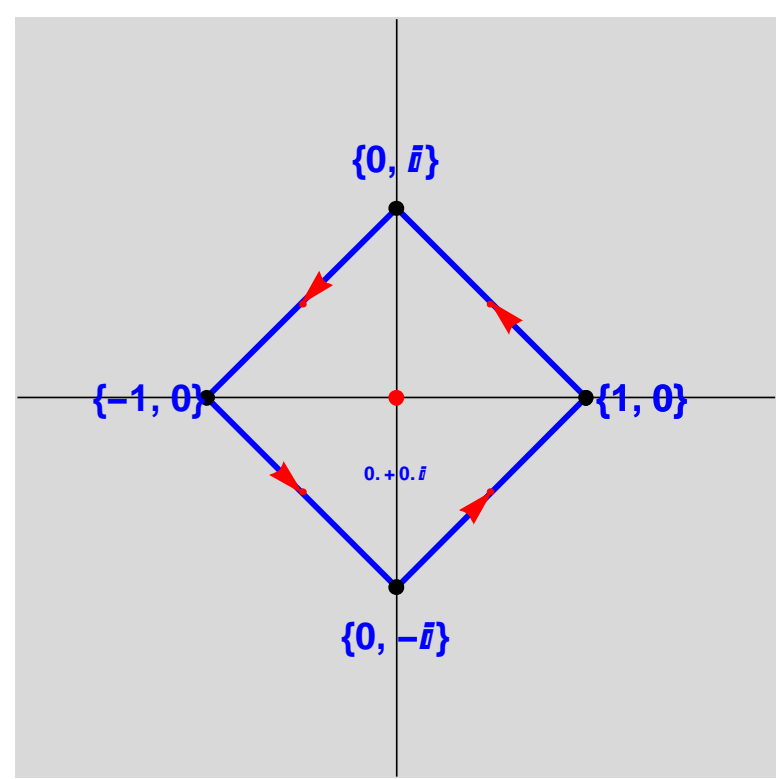

With the interpretation rule defined above the numerical value of the contour integral $\frac{1}{2 \pi i} \oint_{\gamma} \frac{e^{z}}{z} d z$ with the contour $\gamma$ is easily computed as $\frac{1}{2 \pi \text { in }} \oint_{\gamma 1} \frac{e^{z}}{z} d z / /$ ratchop

1 
(ii) Furthermore, the number of zeros of an analytic function $f(z)$ (counting repeated roots) within the closed contour $\gamma$ can be determined by a contour integral $n=\frac{1}{2 \pi i} \oint_{\gamma} \frac{f^{\prime}(z)}{f(z)} d z$ too :

$\mathcal{P}\left[z_{-}\right]:=z^{5}-\frac{z^{3}}{2}-z^{2}+\frac{1}{2} ; \quad \rho=z /$. Solve $[\mathcal{P}[z]=0, z]$

cmplxPts $=(\# \llbracket 1 \rrbracket+\dot{i} \# \llbracket 2 \rrbracket) \& / @\left(\{\operatorname{Re}[\rho], \operatorname{Im}[\rho]\}^{\top}\right)$;

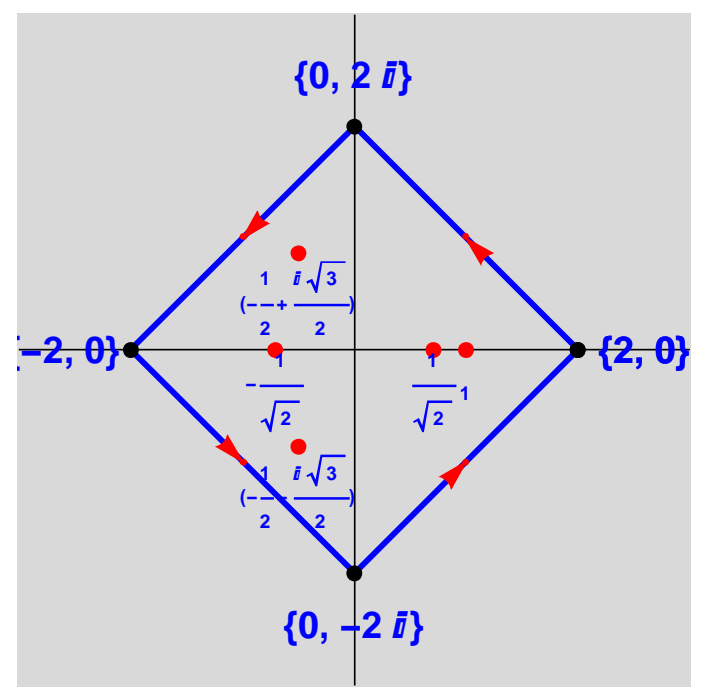

Hence, the contour integral confirms that 5 roots of $\mathcal{P}(\mathbf{z})$ are enclosed by the contour $\gamma 2$ :

$\left(\frac{1}{2 \pi i} \oint_{\gamma 2} \frac{\mathcal{P}^{\prime}[z]}{\mathcal{P}[z]} d z\right) / /$ Chop//Round

5 
(iii) Moreover, contour integrals can also be evaluated numerically over parametricallydefined paths utilizing the notation :

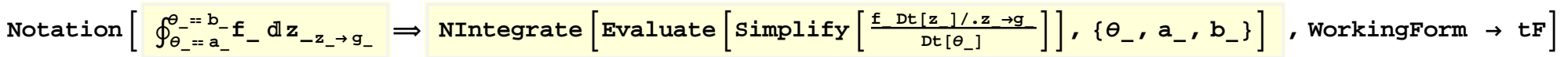

Total differentiation $\mathbf{D t}$ is used for change of variables. For example, a circle in the complex plane can be parameterized by $\boldsymbol{r} \boldsymbol{e}^{\boldsymbol{i} \boldsymbol{\theta}}$ with radius 2 :

$\mathcal{P}\left[z_{-}\right]:=z^{5}-\frac{z^{3}}{2}-z^{2}+\frac{1}{2} ; \quad \rho=z /$. Solve $[\mathcal{P}[z]=0, z] ;$

CmplxPts $=(\# \llbracket 1 \rrbracket+\dot{i} \# \llbracket 2 \rrbracket) \& / @\left(\{\operatorname{Re}[\rho], \operatorname{Im}[\rho]\}^{\top}\right)$;

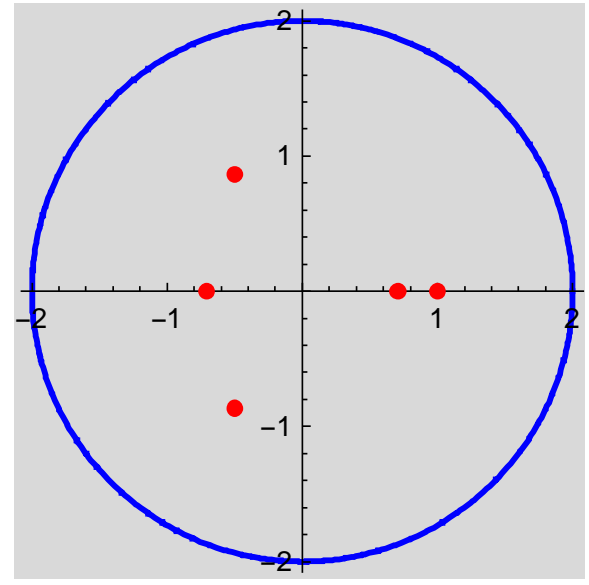

The integral of $\frac{\mathcal{P}^{\prime}[z]}{\mathcal{P}[z]}$ along this circular contour again confirms the previous result that there are 5 zeros inside the contour :

$\frac{1}{2 \pi i} \oint_{\theta=0}^{\theta==2 \pi} \frac{\mathcal{P}^{\prime}[z]}{\mathcal{P}[z]} d z_{z \rightarrow 2 e^{i \theta}} / /$ Round 
The integral of $\frac{1}{z}$ along the same circular contour is :

$\frac{1}{2 \pi i} \oint_{\theta=0}^{\theta=2 \pi} \frac{1}{z} d z_{z \rightarrow 2} e^{i \theta} / /$ Round

Note : machine complex numbers have machine reals for both real and imaginary parts. Consequently, Chop does not make the real part of machine complex numbers an exact zero.

Here is a more complicated spiral-like contour $\boldsymbol{p}(\boldsymbol{\theta})=\left(\boldsymbol{\theta} \boldsymbol{e}^{\boldsymbol{i} \boldsymbol{\theta}}+\mathbf{1}\right)$ :

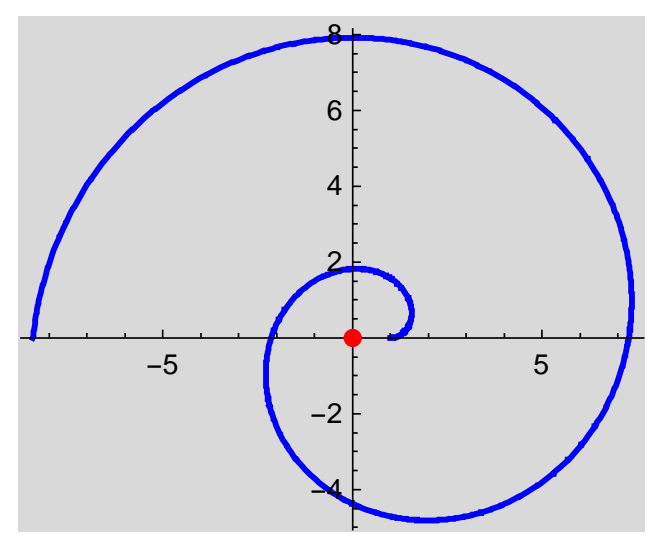

with the associated contour integral

$\oint_{\theta=0}^{\theta==3 \pi} \frac{1}{z} d z_{z \rightarrow \theta e^{i \theta}+1}$ 
(iv) Finally, line integrals [2] arise for scalar or vector fields taking the form $\int_{\mathcal{L}} \boldsymbol{F}(\boldsymbol{x}) \cdot d \boldsymbol{x}$, where $\mathcal{L}$ denotes the integration path. In three dimensions, an explicit representation is

$$
\int_{\mathcal{L}} \vec{F}(\vec{R}) \cdot d \vec{R}=\int_{\mathcal{L}} F_{1}(x, y, z) d x+F_{2}(x, y, z) d y+F_{3}(x, y, z) d z
$$

here $\vec{R}=\{\boldsymbol{x}, \boldsymbol{y}, \boldsymbol{z}\}$ are the coordinates along the path $\mathcal{L}$.

One way of dealing with line integrals is to parameterize the path $\mathcal{L}$ by specifying the coordinates in terms of a parameter $t$ for example $x=f_{1}(t)$, $y=f_{2}(t), z=f_{3}(t)$ in addition to the range of $t$ - values for the desired path $\mathcal{L}$. A direct implementation is given in reference [3] .

A possible construct for LineIntegral is given by (due to private communication with Paul Abbott, Nov. 2015)

\section{? LineIntegral}

Linelntegral[ $F_{1} \operatorname{Dt}\left[x_{1}\right]+F_{2} \operatorname{Dt}\left[x_{2}\right]+F_{3} \operatorname{Dt}\left[x_{3}\right],\left\{x_{1}==f_{1}[t], x_{2}==f_{2}[t], x_{3}==f_{3}[t], \ldots, x_{n}==f_{n}[t]\right\}$,

$\left.\{t, a, b\},\left\{x_{1}, x_{2}, x_{3}, \ldots, x_{n}\right\}\right]$ attempts to calculate the line integral in $\left\{x_{1}, x_{2}, x_{3}, \ldots, x_{n}\right\}$ space, by parametrizing the

integration path $\mathcal{L}$ with respect to $t$, from $t=a$ to $t=b$ such that $\mathcal{L}[t]=\left\{x_{1}==f_{1}[t], x_{2}==f_{2}[t], x_{3}==f_{3}[t], \ldots, x_{n}==f_{n}[t]\right\}$,

LineIntegral $\left[f:\right.$ Literal $\left.\left[{ }_{-} D t\left[_{-}\right] \mid+\left({ }_{-} D t\left[_{-}\right] \ldots\right)\right], \mathcal{L}_{-}, p:\left\{t_{-}, a_{-}, b_{-}\right\}, \operatorname{vars}:\left\{_{--}\right\}\right]:=$

$\int_{a}^{b}$ Expand $[(f / . \operatorname{Thread}[\operatorname{vars} \rightarrow \mathcal{L}]) / \operatorname{Dt}[t]] d t /$; Length $[\mathcal{L}]===$ Length $[\operatorname{vars}]$

The pattern $\mathbf{f}:$ Literal [_Dt [_] $\mid+($ Dt [_] .. ) ] uses Alternatives (i.e. $\mid$ ) to test whether the argument corresponds to a one-dimensional line integral $\int \mathbf{F}(\mathbf{x}) \mathbf{d} \mathbf{x} \mathbf{x}$ or to a multi-dimensional line integral of the form $\int \mathbf{F}_{\mathbf{1}}\left(\mathbf{x}_{1}, \mathbf{x}_{\mathbf{2}}, \ldots\right) \boldsymbol{d} \mathbf{l} \mathbf{x}_{\mathbf{1}}+\mathbf{F}_{\mathbf{2}}\left(\mathbf{x}_{\mathbf{1}}, \mathbf{x}_{\mathbf{2}}, \ldots\right) \boldsymbol{d} \mathbf{\mathbf { x } _ { 2 }}+\ldots$. The use of $\mathbf{D t}[\boldsymbol{x}]$ ensures that the appropriate change of variables takes place automatically such as :

$\operatorname{Dt}[x] / . x \rightarrow \operatorname{Sin}[2 \theta]$ 
The integration path $\mathcal{L}$ may be specified by the coordinates $\left\{x_{1}, x_{2}, \ldots, x_{n}\right\}$ of points along the path in terms of a parametert: $\left\{x_{1}==f_{1}(t), x_{2}==f_{2}(t)\right.$, $\left.\ldots, x_{n}=f_{n}(t)\right\}$ together with the parameter range for $t \in\{\mathbf{a}, \mathbf{b}\}$ defining the path $\mathcal{L}$.

Subsequently, two examples of line integrals w.r.t. the following vector functions $f_{i}$ are discussed :

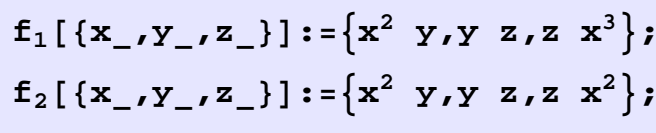

along the path $\mathcal{L}_{i}$ (which must not necessarily be a closed contour, see $\mathcal{L}_{2}$ ):

$\mathcal{L}_{1}\left[t_{-}\right]=\{\operatorname{Cos}[3 t], \operatorname{Sin}[t], \operatorname{Sin}[2 t]\}$

$\mathcal{L}_{2}\left[t_{-}\right]=\{\operatorname{Sin}[t], \operatorname{Cos}[t], t / 5\} ;$

Visualizing the two parametric paths $\mathcal{L}_{i}(\mathbf{t})$ is easy:

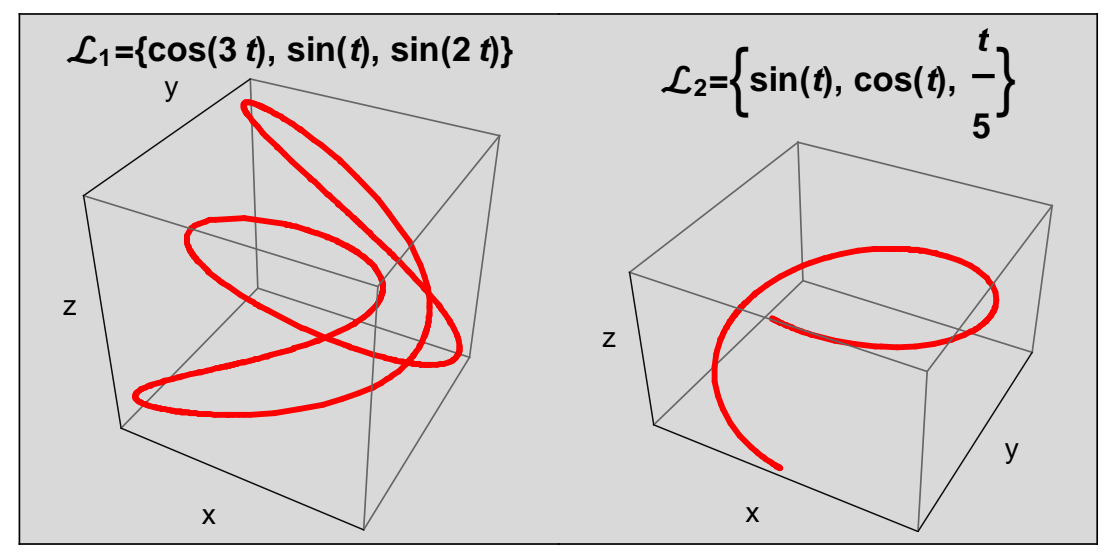

Defining the Cartesian variables $\mathbf{R}$ (or $\rho$ ) and the interval $\mathbf{T}$ (or $\tau$ ) for parametrization then the line integrals turn out to be : 
$\mathbf{z}=$. ;

$R=\{x, y, z\} ; T=\{t, 0,2 \pi\} ;$

$\rho=\{\xi, \eta, \zeta\} ; \tau=\{t,-\pi, \pi\}$;

$\left\{f_{1}[R] . \operatorname{Dt}[R], \mathcal{L}_{1}[t], R, T\right.$, LineIntegral $\left.\left[f_{1}[R] . D t[R], \mathcal{L}_{1}[t],\{t, 0,2 \pi\}, R\right]\right\}$

$\left\{f_{2}[\rho] . \operatorname{Dt}[\rho], \mathcal{L}_{2}[t], \rho, \tau, \quad\right.$ LineIntegral $\left.\left[f_{2}[\rho] . \operatorname{Dt}[\rho], \mathcal{L}_{2}[t],\{t,-\pi, \pi\}, \rho\right]\right\}$

$\left\{x^{2} y \operatorname{Dt}[x]+y z D t[y]+x^{3} z \operatorname{Dt}[z],\{\operatorname{Cos}[3 t], \operatorname{Sin}[t], \operatorname{Sin}[2 t]\},\{x, y, z\},\{t, 0,2 \pi\}, \frac{\pi}{2}\right\}$

$\left\{\zeta \xi^{2} \operatorname{Dt}[\zeta]+\zeta \eta \operatorname{Dt}[\eta]+\eta \xi^{2} \operatorname{Dt}[\xi],\left\{\operatorname{Sin}[t], \operatorname{Cos}[t], \frac{t}{5}\right\},\{\xi, \eta, \zeta\},\{t,-\pi, \pi\}, \frac{7 \pi}{20}\right\}$

A possible notation $\int_{\mathcal{L}, \mathbf{p}} \mathbf{f} \cdot \boldsymbol{d} \mathbf{t} \mathbf{t}[\mathbf{r}]$ is :

$\int_{\mathcal{L}_{-}, \mathbf{p}_{-}} \mathbf{f}_{-} \cdot d \mathbf{d t}\left[\mathbf{r}_{-}\right] \Leftrightarrow$ LineIntegral $\left[\mathbf{f}_{-} \cdot \mathrm{Dt}\left[\mathbf{r}_{-}\right], \mathcal{L}_{-}, \mathbf{P}_{-}, \mathbf{r}_{-}\right]$

with which the same results as above are obtained for the line integrals :

$$
\begin{aligned}
& \left\{\int_{\mathcal{L}_{1}[t], T} f_{1}[R] \cdot d t[R], \int_{\mathcal{L}_{2}[t], \tau} f_{2}[\rho] \cdot d t[\rho]\right\} \\
& \left\{\frac{\pi}{2}, \frac{7 \pi}{20}\right\}
\end{aligned}
$$




\section{- Residues}

\section{- Introductory remarks}

The residue theorem in complex analysis is a powerful tool for the calculation of integrals involving complex functions $\boldsymbol{f}(\boldsymbol{z})$ with $\mathbf{z} \in \mathbb{C}$; in many cases it will also be used for the evaluation of real integrals (with real variables $\mathbf{x} \in \mathbb{R}$ ) as well [5-12].

As an extension of the so-called Cauchy integral theorem the residue theorem is a method for calculating contour integrals of type $\oint_{\gamma} f(z) d z$ where $\gamma$ denotes a positive-oriented closed curve in the complex plane $\mathbb{C}$. The integrand $\boldsymbol{f}(\boldsymbol{z})$ is an analytic function except for a finite number of isolaled singular points $\left\{z_{1}, z_{2}, \ldots z_{n}\right\}$ inside the closed contour $\gamma$. Thus

$$
\oint_{\gamma} f(\zeta) d \zeta=\left.2 \pi i \sum_{k=1}^{m} w\left(\gamma, z_{k}\right) \operatorname{Res} f(z)\right|_{z=z_{k}}
$$

where $\boldsymbol{w}\left(\gamma, z_{k}\right)$ denotes the winding number of the curve $\gamma$ w.r.t. the point $z_{k}$. It is an integer $\boldsymbol{n} \in \mathbb{N}$ which intuitively measures how many times the curve $\gamma$ winds around the point $z_{k}$; it is positive $\boldsymbol{n}>\mathbf{0}$ if $\gamma$ winds in a counter-clockwise manner around $z_{k}$ and 0 if $\gamma$ does not move around $z_{k}$ at all. If $\gamma$ is a positively oriented Jordan curve, then $w\left(\gamma, z_{k}\right)=\mathbf{1}$.

If $f(z)$ has a pole of order $\boldsymbol{m}$ in $z_{0}$ then $g(z)=\left(z-z_{0}\right)^{m} f(z)$ again is analytic in $z_{0}$. For a simple closed contour $\gamma$ which contains only $z_{0}$ but no other singular points of $f(z)$ then

$$
\oint_{\gamma} f(\zeta) d \zeta=\oint_{\gamma} \frac{g(\zeta)}{\left(\zeta-z_{0}\right)^{m}} d \zeta=2 \pi i \frac{g^{(m-1)}\left(z_{0}\right)}{(m-1) !}=2 \pi i \frac{1}{(m-1) !} \lim _{z \rightarrow z_{0}} \frac{d^{m-1}}{d z^{m-1}}\left(\left(z-z_{0}\right)^{m} f(z)\right)=\left.2 \pi i \operatorname{Res} f(z)\right|_{z=z_{0}}
$$

The residue is just the coefficient $\boldsymbol{a}_{-1}$ with $\boldsymbol{n}=\mathbf{- 1}$ in the Laurent series of the complex function

$$
f(z)=\sum_{k=1}^{\infty} \frac{a_{-k}}{\left(z-z_{0}\right)^{k}}+\sum_{k=0}^{\infty} a_{k}\left(z-z_{0}\right)^{k} \text { with coefficients } a_{k}=\frac{1}{2 \pi i} \int_{\mathcal{K}\left(z_{0}\right)} \frac{f(\zeta)}{\left(\zeta-z_{0}\right)^{k+1}} d \zeta
$$


where $\mathcal{K}\left(z_{0}\right)$ is a positive oriented annulus around $z_{\mathbf{0}}$. The function $\boldsymbol{f}(\boldsymbol{z})$ is holomorph except for $\boldsymbol{z}_{\mathbf{0}}$.

Mathematica provides the built-in procedure Residue for calculating residues

\section{? Residue}

Residue $\left[\operatorname{expr},\left\{z, z_{0}\right\}\right]$ finds the residue of $\operatorname{expr}$ at the point $z=z_{0} . \gg$

with the singular point $z_{0}$ to be given. However, it is more convenient to make use of the user-defined procedure calculateResidue which determines all or a subset of poles $z_{i}$ for the function $f(z)$ given. The poles $z_{i}$ and their multiplicities $\mu_{i}$ are determined internally by the procedure polesOfComplexFunction and multiplicityofPoles. Then, the residues for single or multiple poles are calculated according to the value of the parameter selectPoles which could either be All or $\{\ldots, n, \ldots m, \ldots\}$.

\section{? calculateResidues}

calculateResidues[f, z, selectPoles_All, polesRange_\{ \}, onoff_"Off"] evaluates the residues symbolically. 'f' denotes the function $f(z)$ with ' $z$ ' as complex variable $z \in \mathbb{C}$. The poles of the denominator of $f(z)$ are calculated using the procedure polesOfComplexFunction[f,z,selectPoles,polesRange,onoff] which returns the list of lists $\{$ poles, $\mu$,solK\}. 'poles' contains the list of poles (which are determined either by Reduce or Solve), ' $\mu$ ' is a list containing the multiplicity of every pole. 'solK' assumes the value T|F. With the parameter 'selectPoles' $=\{i, j, \ldots\}$ a subset of poles can be selected for the final calculation of residues. Default value for 'selectPoles' is All. With an additional global variable $\$ k$ the number of poles can be controlled. For denominators e.g. $\left(e^{z}-1\right)==0$ a sequence of poles results such as $z_{k}=2 \pi i \cdot k$ with an additional index $k \in \mathbb{N}$. Hence, for $\$ k=2$ the index $k$ assumes the values $\{0,1,2\}$ whereas for $\$ k=-2$ the values $k=\{-2,-1,0,1,2\}$. The sum of residues for the poles selected is calculated and returned. With onoff= "No" printout is completely suppressed.

In order to evaluate real-valued integrals, e.g. $\int_{-\infty}^{+\infty} f(x) d x$, one has to proceed as follows : the integrand $f(x)$ must be extended into the complex plane and the residues be computed where the part of the real axis is completed to a closed curve by adding a half-circle in the upper or lower half-plane. Then the integral over this closed contour can be computed by means of the residue theorem. Often, the semi-circle part of the integral tends to zero as the radius (of the semi-circle) $\mathbf{R} \rightarrow \infty$ so that only that part of the integral along the real-axis remains. 
Subsequently, 20 examples of residues are given. They comprise the following classes of functions :

- Residues of rational functions $f(z)=\frac{p(z)}{q(z)}$

Example 1: Residue of $f(z)=\frac{1}{z}:\left.\quad \operatorname{Res} f(z)\right|_{z=0}=1$

Example 2 : Residue of $f(z)=\frac{z^{2}-2 z+7}{z-2}: \quad$ Res $\left.f(z)\right|_{z=2}=7$

$f(z)=\frac{z^{2}-2 z+7}{z-2}=\frac{7}{z-2}+2(z-2)^{0}+1(z-2)$ has in $z_{0}=2$ the residue Res $\left.f(z)\right|_{z=2}=7$ as can easily be shown with the procedure Residue

Residue $\left[\frac{z^{2}-2 z+7}{z-2},\{z, 2\}\right]$

7

calculateResidues $\left[\frac{z^{2}-2 z+7}{z-2}, z, A l l,\{\}, " O n "\right]$

Sorted poles of $\mathrm{f}(\mathrm{z})=\frac{z^{2}-2 z+7}{z-2}:\{2\}$

$z_{1}=2$ is a 1 -fold pole

For rational functions $f(z)=\frac{z^{2}-2 z+7}{z-2} ;\{$ poles, $\mu\}:\{\{2\},\{1\}\}$; solK $=F$ 


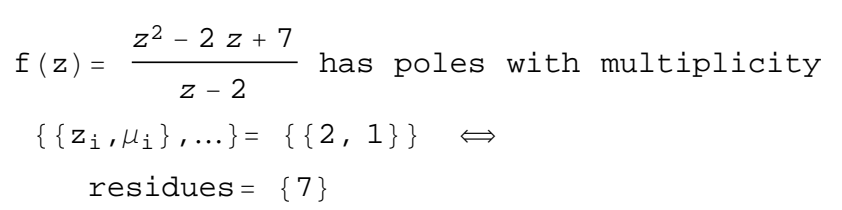

7

Example 3 : Residue of $f(z)=\frac{1}{z^{2}}: \quad \quad$ Res $\left.f(z)\right|_{z=0}=0$

Example 4 : Residue of $f(z)=\frac{1}{1+z^{2}}:\left.\quad \operatorname{Res} f(z)\right|_{z= \pm i}=\mp \frac{i}{2}$

Example 5 : Residue of $f(z)=(z-\alpha)^{m}: \quad$ Res $\left.f(z)\right|_{z=\alpha}=1$ (only for $m=-1$ )

Example 6 : Residue of $f(z)=\frac{1}{\left(z^{2}-2\right)}: \quad$ Res $\left.f(z)\right|_{z= \pm 2}= \pm \frac{1}{4}$

Example 7 : Residue of $f(z)=\frac{1}{z(z-i)^{2}}:\left.\quad \operatorname{Res} f(z)\right|_{z=i}=1$

Example 8 : Residue of $f(z)=\frac{2 z^{2}+z+1}{\left(z^{2}+1\right)(z-2 i)^{2}}:\left.\operatorname{Res} f(z)\right|_{z=\{i, 2 i\}}=\frac{1-i}{18}$

The function $f(z)=\frac{2 z^{2}+z+1}{\left(z^{2}+1\right)(z-2 i)^{2}}$ has a single pole at $\mathbf{z}= \pm \boldsymbol{i}$ and a double pole at $\mathbf{z}=\mathbf{2 i}$. Thus the residues for these three poles are given as $\mathbf{z} 0=\{-\dot{i}, \dot{i}, 2$ ì $\}$

res $=\left(\operatorname{Residue}\left[\frac{2 z^{2}+z+1}{\left(z^{2}+1\right)(z-2 \dot{i})^{2}},\{z, \#\}\right] / @\{-\dot{i}, \dot{i}, 2 \dot{i}\}\right) / /$ Together 
$\left\{-\frac{1}{18}+\frac{\dot{1}}{18},-\frac{1}{2}-\frac{\dot{1}}{2}, \frac{5}{9}+\frac{4 \dot{1}}{9}\right\}$

If all three poles are considered the sum of residues vanishes; however, if only poles $z_{\mathbf{2}}$ and $z_{\mathbf{3}}$ in $\boldsymbol{H}_{+}$are taken into account then one obtains

\{res /. \{List $\rightarrow$ Plus\}, (* sum all 3 residues *)

res $\llbracket\{2,3\} \rrbracket /$. List $\rightarrow$ Plus $\}\}$ (* sum residues $2,3 *$ )

$\left\{0, \frac{1}{18}-\frac{\dot{1}}{18}\right\}$

The same result is obtained with calculateResidues

calculateResidues $\left[\frac{2 z^{2}+z+1}{\left(z^{2}+1\right)(z-2 i)^{2}}, z,\{2,3\},\{\}, " O n "\right]$

Sorted poles of $f(z)=\frac{2 z^{2}+z+1}{(z-2 \dot{i})^{2}\left(z^{2}+1\right)}:\{-\dot{\mathbb{1}}, \dot{\mathbb{1}}, 2 \dot{\mathbb{1}}, 2 \dot{\mathbf{i}}\}$

$z_{1}=-i$ is a 1 -fold pole

$\mathrm{z}_{2}=\dot{\mathrm{i}}$ is a 1 -fold pole

$z_{3}=2$ i is a 2 -fold pole 
For rational functions $f(z)=\frac{2 z^{2}+z+1}{(z-2 \dot{i})^{2}\left(z^{2}+1\right)} ;\{$ poles,$\mu\}:\{\{-\dot{1}, \dot{1}, 2 \dot{\mathbb{1}}\},\{1,1,2\}\}$; solK=F

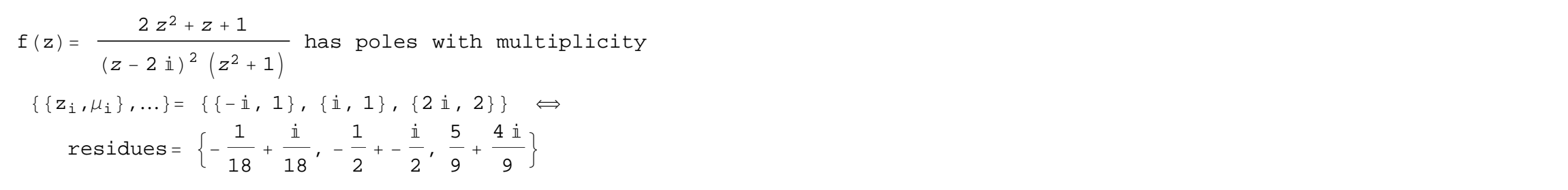

Residues $\left.\sum_{i} \operatorname{Res}(f(z))\right|_{z=z_{i}}=\frac{1}{18}-\frac{\dot{1}}{18}$ for $\operatorname{pole}(s) \quad i=\{2,3\}$

$\frac{1}{18}-\frac{\dot{1}}{18}$

Example 9 : Residue of $f(z)=\frac{2 z^{2}+17}{(z-1)^{3}(z+1)^{3}}:\left.\operatorname{Res} f(z)\right|_{z=+1}=\frac{49}{16}$

The function $f(z)=\frac{\mathbf{2} z^{2}+17}{(z-1)^{3}(z+1)^{3}}$ has in $\mathbf{z}= \pm \mathbf{1}$ a pole of 3rd order. The corresponding Laurent expansion for $f(z)$ can be calculated straightforward with

$f\left[z_{-}\right]:=\frac{2 z^{2}+17}{(z-1)^{3}(z+1)^{3}}$

$(\operatorname{ser}=(\operatorname{Series}[f[z],\{z, 1,1\}] / /$ Normal $) /.\{(z-1) \rightarrow \zeta\} / .\{\zeta \rightarrow$ HoldForm $[(z-1)]\}) / /$ polyForm

$a_{1}=$ Coefficient $[$ ser //ReleaseHold, $1 /(z-1)]$

$\frac{253(z-1)}{128}-\frac{83}{32}+\frac{49}{16(z-1)}-\frac{49}{16(z-1)^{2}}+\frac{19}{8(z-1)^{3}}$ 
and the residue is obtained as coefficient of $(z-\mathbf{1})^{\mathbf{- 1}}$ of the Laurent series.

The same result is obtained with calculateResidues for the 3 -fold pole $z_{2}=\mathbf{1}$

calculateResidues $\left[\frac{2 z^{2}+17}{(z-1)^{3}(z+1)^{3}}, z,\{2\},\{\}, " O n "\right]$

Sorted poles of $f(z)=\frac{2 z^{2}+17}{\left(z^{2}-1\right)^{3}}:\{-1,-1,-1,1,1,1\}$

$z_{1}=-1$ is a 3 -fold pole

$z_{2}=1$ is a 3 -fold pole

For rational functions $f(z)=\frac{2 z^{2}+17}{(z-1)^{3}(z+1)^{3}} ;$ poles, $\left.\mu\right\}:\{\{-1,1\},\{3,3\}\}$; solK $=F$

$f(z)=\frac{2 z^{2}+17}{(z-1)^{3}(z+1)^{3}}$ has poles with multiplicity

$\left\{\left\{z_{i}, \mu_{i}\right\}, \ldots\right\}=\{\{-1,3\},\{1,3\}\} \Leftrightarrow$

residues $=\left\{-\frac{49}{16}, \frac{49}{16}\right\}$ 
$\left.\operatorname{Residues} \sum_{i} \operatorname{Res}(f(z))\right|_{z=z_{i}}=\frac{49}{16}$ for $\operatorname{pole}(s) \quad i=\{2\}$

49

Example $10:$ Residue of $f(z)=\frac{z^{2}}{\left(z^{4}+1\right)}: \quad \Sigma$ Res $\left.f(z)\right|_{z=\frac{ \pm 1+i}{\sqrt{2}}}=\frac{1}{2 \sqrt{2} i}$

Example 11 : Residue of $f(z)=\frac{1}{\left(1+z^{2}\right)^{n}}: \quad$ Res $\left.\frac{1}{\left(1+z^{2}\right)^{n}}\right|_{z=i}=\frac{1}{i} \frac{1}{2^{2 n-1}}\left(\begin{array}{c}2 n-2 \\ n-1\end{array}\right)$

The residue for $z_{0}=\boldsymbol{i}$ is :

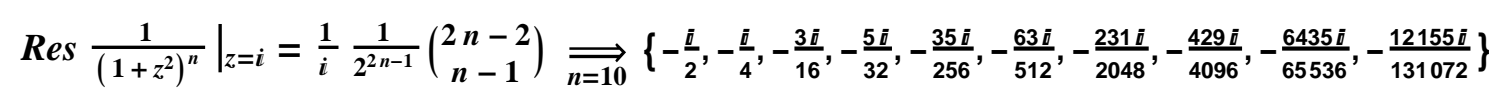

as can be easily shown with Mathematica.

\$Assumptions $=(\mathrm{n} \in$ Integers $)$;

$t_{1}=$ Table $\left[\right.$ Residue $\left[\frac{1}{\left(1+z^{2}\right)^{n}},\{z, \dot{i n}\},\{n, 1,10\}\right]$

$t_{2}=\operatorname{Table}\left[\frac{1}{i} \frac{1}{2^{2 n-1}}\left(\begin{array}{c}2 n-2 \\ n-1\end{array}\right),\{n, 1,10\}\right]$;

$t_{1}==t_{2}$

$\left\{-\frac{\dot{i}}{2},-\frac{\dot{i}}{4},-\frac{3 \dot{i}}{16},-\frac{5 \dot{i}}{32},-\frac{35 \dot{i}}{256},-\frac{63 \dot{i}}{512},-\frac{231 \dot{i}}{2048},-\frac{429 \dot{i}}{4096},-\frac{6435 \dot{i}}{65536},-\frac{12155 \dot{i}}{131072}\right\}$

True

The same series of residues is obtained with calculateResidues . 
Table $\left[\right.$ calculateResidues $\left[\frac{1}{\left(1+\mathbf{z}^{2}\right)^{\mathbf{n}}}, \mathbf{z},\{2\},\{\}, "\right.$ No" $\left.],\{\mathrm{n}, 1,10\}\right]$

$\left\{-\frac{\dot{i}}{2},-\frac{\dot{i}}{4},-\frac{3 \dot{i}}{16},-\frac{5 \dot{i}}{32},-\frac{35 \dot{i}}{256},-\frac{63 \dot{i}}{512},-\frac{231 \dot{i}}{2048},-\frac{429 \dot{i}}{4096},-\frac{6435 \dot{i}}{65536},-\frac{12155 \dot{i}}{131072}\right\}$

Example 12 : Residues of $f_{n}(z)=\frac{g(z)}{z^{n}}:\left.\quad \operatorname{Res} f_{n}(z)\right|_{z=0}=\frac{1}{n !} g^{(n)}(0) \quad(n=0,1,2, \ldots)$

Example 13 : Residue of $f(z)=e^{1 / z}:\left.\quad \operatorname{Res} f(z)\right|_{z=0}=1$

Because the function $f(z)=e^{1 / z}$ has an essential singularity in $z_{0}=0$, hence immediate application of Residue gives no answer.

However, the Laurent expansion of $e^{1 / z}$ for $z_{0}=\mathbf{0} f(z)=\sum_{k=0}^{\infty} \frac{1}{k !}\left(\frac{1}{z}\right)^{k}=\sum_{k=-\infty}^{0} \frac{1}{(-k) !} z^{k}$ provides the following series expanded up to $n$ terms :

$\operatorname{truncSer}\left[\mathbf{n}_{-}\right]:=\left(\operatorname{Series}\left[\left(e^{1 / \mathbf{z}} / .\{1 / \mathbf{z} \rightarrow \zeta\}\right),\{\zeta, 0, \mathbf{n}\}\right] / /\right.$ Normal $) / .\{\zeta \rightarrow 1 / \mathbf{z}\}$

(ser $=$ truncSer $[8]) / /$ polyForm

$1+\frac{1}{z}+\frac{1}{2 z^{2}}+\frac{1}{6 z^{3}}+\frac{1}{24 z^{4}}+\frac{1}{120 z^{5}}+\frac{1}{720 z^{6}}+\frac{1}{5040 z^{7}}+\frac{1}{40320 z^{8}}$

Obviously, for the truncated series the residue assumes the correct value $\mathbf{a}_{-\mathbf{1}}=\left.\operatorname{Res} \mathrm{e}^{\mathbf{1 / z}}\right|_{z=\mathbf{0}}=\mathbf{1}$

Residue [truncSer $[100],\{z, 0\}]$

1

- Residues for products of rational and trigonometric functions $f(z)=\frac{p(z)}{q(z)} \cdot\left\{\begin{array}{l}\cos (\alpha z) \\ \sin (\alpha z)\end{array}\right.$

Example $14:$ Residues of $f(z)=\frac{e^{i z}}{1+z^{4}}:\left.\quad \operatorname{Res} f(z)\right|_{z=( \pm 1+i) / \sqrt{2}}=-\frac{i e^{-\frac{1}{\sqrt{2}}}\left(\operatorname{Cos}\left[\frac{1}{\sqrt{2}}\right]+\operatorname{Sin}\left[\frac{1}{\sqrt{2}}\right]\right)}{2 \sqrt{2}}$ 
The poles of $f(z)=\frac{1}{1+z^{4}}$ located in the upper half-plane $H_{+}$are $z_{3,4}=\frac{ \pm 1+i}{\sqrt{2}}$ as determined by means of sol = Solve $\left[1+z^{4}==0, z\right] /$ Rule $\left.\left[a_{-}, b_{-}\right] \rightarrow b\right\} / /$ Flatten $/ / c e$;

sols = SortBy $[$ sol, $0<\operatorname{Im}[\#] \&]$

$\operatorname{sols} \llbracket\{3,4\} \rrbracket$

$\left\{-\frac{1+\dot{i}}{\sqrt{2}}, \frac{1-\dot{i}}{\sqrt{2}},-\frac{1-\dot{i}}{\sqrt{2}}, \frac{1+\dot{\mathbb{1}}}{\sqrt{2}}\right\}$

$\left\{-\frac{1-\dot{\mathbb{1}}}{\sqrt{2}}, \frac{1+\dot{\mathbb{1}}}{\sqrt{2}}\right\}$

Then, the associated residues are obtained as $\frac{(\mp 1-\dot{r})}{4 \sqrt{2}} e^{-\frac{(1 \mp \dot{i})}{\sqrt{2}}}$

$\left(\operatorname{Residue}\left[\frac{e^{\dot{i} z}}{1+z^{4}},\{z, \#\}\right] /\right.$ sols $\left.\llbracket\{3,4\} \rrbracket\right) / .\{$ List $\rightarrow$ Plus $\} / /$ ce $/ /$ sf

$-\frac{i e^{-\frac{1}{\sqrt{2}}}\left(\operatorname{Cos}\left[\frac{1}{\sqrt{2}}\right]+\operatorname{Sin}\left[\frac{1}{\sqrt{2}}\right]\right)}{2 \sqrt{2}}$

The same result is achieved with calculateResidues

calculateResidues $\left[\frac{e^{\text {i z }}}{1+z^{4}}, z,\{3,4\}\right] / /$ ce // sf 
$f(z)=\frac{\mathbb{e}^{i z}}{z^{4}+1}$ has poles with multiplicity

$\left\{\left\{z_{i}, \mu_{i}\right\}, \ldots\right\}=\left\{\left\{-(-1)^{1 / 4}, 1\right\},\left\{-(-1)^{3 / 4}, 1\right\},\left\{(-1)^{1 / 4}, 1\right\},\left\{(-1)^{3 / 4}, 1\right\}\right\} \Leftrightarrow$

residues $=\left\{\frac{\left(\frac{1}{4}+\frac{\dot{i}}{4}\right) e^{\frac{1}{\sqrt{2}}}\left(\operatorname{Cos}\left[\frac{1}{\sqrt{2}}\right]+-\dot{i} \operatorname{Sin}\left[\frac{1}{\sqrt{2}}\right]\right)}{\sqrt{2}},-\frac{\left(\frac{1}{4}+-\frac{\dot{i}}{4}\right) e^{\frac{1}{\sqrt{2}}}\left(\operatorname{Cos}\left[\frac{1}{\sqrt{2}}\right]+\dot{i} \sin \left[\frac{1}{\sqrt{2}}\right]\right)}{\sqrt{2}}\right.$,

$\left.\frac{\left(\frac{1}{4}+\frac{\dot{i}}{4}\right) e^{-\frac{1}{\sqrt{2}}}\left(\operatorname{Cos}\left[\frac{1}{\sqrt{2}}\right]+\dot{i} \operatorname{Sin}\left[\frac{1}{\sqrt{2}}\right]\right)}{\sqrt{2}}, \frac{\left(\frac{1}{4}+-\frac{\dot{i}}{4}\right) e^{-\frac{1}{\sqrt{2}}}\left(\operatorname{Cos}\left[\frac{1}{\sqrt{2}}\right]+-\dot{i} \operatorname{Sin}\left[\frac{1}{\sqrt{2}}\right]\right)}{\sqrt{2}}\right\}$

$\left.\operatorname{Residues} \sum_{i} \operatorname{Res}(f(z))\right|_{z=z_{i}}=-\frac{\dot{i} e^{-\frac{1}{\sqrt{2}}}\left(\sin \left(\frac{1}{\sqrt{2}}\right)+\cos \left(\frac{1}{\sqrt{2}}\right)\right)}{2 \sqrt{2}}$ for $\operatorname{pole}(s) \quad i=\{3,4\}$

$-\frac{i e^{-\frac{1}{\sqrt{2}}}\left(\operatorname{Cos}\left[\frac{1}{\sqrt{2}}\right]+\operatorname{Sin}\left[\frac{1}{\sqrt{2}}\right]\right)}{2 \sqrt{2}}$

Example 15 : Residues of $f(z)=\frac{1}{\sin (1 / z)}:\left.\quad \operatorname{Res} f(z)\right|_{z=0}=\frac{1}{6}$

Example 16 : Residues of $f(z)=\frac{1}{\sin (z)^{n}}:\left.\quad \operatorname{Res} \frac{1}{\sin (z)^{n}}\right|_{z=0}=\frac{\text { Pochhammer }\left[\frac{1}{2},-1+n\right]}{\text { Pochhammer[ 1,-1+n] }}$

The power series expansion of $f(z)=\sin (z)^{-n}$ for $z=z_{0}$ for order $n=9$ is generated.

$\operatorname{ser} 9=\operatorname{Series}\left[\frac{1}{\operatorname{Sin}[z]^{n}},\left\{z, z_{0}=0, n=9\right\}\right] / /$ Normal 
$\frac{1}{z^{9}}+\frac{3}{2 z^{7}}+\frac{47}{40 z^{5}}+\frac{3229}{5040 z^{3}}+\frac{35}{128 z}+\frac{1295803 z}{13305600}+$

$\frac{1313487619 z^{3}}{43589145600}+\frac{172819963 z^{5}}{20756736000}+\frac{248460536251 z^{7}}{118562476032000}+\frac{1245940242556237 z^{9}}{2554547108585472000}$

Obviously, the residue of $f(z)=\sin (z)^{-n}$ in $z_{0}=0$ is given as the coefficient of $z^{-1}$ of the truncated series :

Coefficient $\left[\operatorname{ser} 9, \mathbf{z}^{-1}\right]$

35

128

Here calculateResidues is applied to the series expansions of $\sin (z)^{-\boldsymbol{n}}$ for $\boldsymbol{n}=\{\mathbf{1}, \ldots \mathbf{2 1}\}$; the series are truncated/reduced to their coefficients $a_{-1}(\mathbf{n}, \mathbf{z})$.

\$Assumptions $=(\mathbf{n} \in$ Integers $)$

$a_{-1}\left[n_{-}, z_{-}\right]:=$Coefficient $\left[\operatorname{Series}\left[\frac{1}{\operatorname{Sin}[z]^{n}},\{z, 0, n\}\right] / /\right.$ Normal, $\left.z^{-1}\right]$

residues $=$ Table $\left[\right.$ calculateResidues $\left.\left[a_{-1}[n, z] * \frac{1}{z}, z,\{1\},\{\}, " N o "\right],\{n, 1,21,2\}\right]$

$\left\{1, \frac{1}{2}, \frac{3}{8}, \frac{5}{16}, \frac{35}{128}, \frac{63}{256}, \frac{231}{1024}, \frac{429}{2048}, \frac{6435}{32768}, \frac{12155}{65536}, \frac{46189}{262144}\right\}$

The sequence of residues of $\sin (z)^{-n}$ in $z_{0}=\mathbf{0}$ can be expressed as quotient of Pochhammer symbols $\frac{(1 / 2)_{m-1}}{(1)_{m-1}}$

resSin2n = FindSequenceFunction[residues] $[\mathrm{m}]$;

ressin2n / t $F$

Table [resSin2n, $\{\mathrm{m}, 1,11\}]$ 
$\frac{\left(\frac{1}{2}\right)_{m-1}}{(1)_{m-1}}$

$\left\{1, \frac{1}{2}, \frac{3}{8}, \frac{5}{16}, \frac{35}{128}, \frac{63}{256}, \frac{231}{1024}, \frac{429}{2048}, \frac{6435}{32768}, \frac{12155}{65536}, \frac{46189}{262144}\right\}$

Some more sophisticated residues are given in the following subsection

- Further residues for functions involving $\zeta(z)$ and $\Gamma(z)$

Example $17:$ Residues of $f_{n}(z)=\frac{\zeta(z)}{(z-1)^{n}}:\left.\operatorname{Res} f_{n}(z)\right|_{z=1}=(-1)^{(n-1)} \frac{\gamma_{n-1}}{(n-1) !} \quad(n=2,3, \ldots)$

For the function $f(z)=\frac{\zeta(z)}{(z-1)^{n}}$ (where $\zeta(\mathbf{z})$ is the Zeta function) the residue for $z_{0}=\mathbf{1}$ is obtained as $(\mathbf{- 1})^{n} \frac{\gamma_{n}}{n !}$ with $\gamma_{n}$ being the buillt-in function Stielt jesGamma $[n]$.

Table $\left[\operatorname{Residue}\left[\frac{\zeta(z)}{(z-1)^{n}},\{z, 1\}\right],\{n, 2,10\}\right] / / t F$

$\left\{-\gamma_{1}, \frac{\gamma_{2}}{2},-\frac{\gamma_{3}}{6}, \frac{\gamma_{4}}{24},-\frac{\gamma_{5}}{120}, \frac{\gamma_{6}}{720},-\frac{\gamma_{7}}{5040}, \frac{\gamma_{8}}{40320},-\frac{\gamma_{9}}{362880}\right\}$

The residue for any power of $(z-\mathbf{1})^{n}$ can be calculated with calculateResidues.

calculateResidues $\left[\frac{\zeta(z)}{(z-1)^{51}}, z,\{1\}\right]$

$f(z)=\frac{\zeta(z)}{(z-1)^{51}}$ has poles with multiplicity

$\left\{\left\{z_{i}, \mu_{i}\right\}, \ldots\right\}=\{\{1,51\}\} \Leftrightarrow$

residues $=\{$ StieltjesGamma [50] / 30414093201713378043612608166064768844377641568960512000000000000$\}$ 
Residues $\left.\sum_{i} \operatorname{Res}(\mathrm{f}(\mathrm{z}))\right|_{\mathrm{z}=\mathrm{z}_{\mathrm{i}}}=$

$\gamma_{50} / 30414093201713378043612608166064768844377641568960512000000000000 \quad$ for pole $(s) \quad i=\{1\}$

Example 18 : Residues of $f(z, m)=\Gamma(z-m) \ldots \Gamma(z-1) \Gamma(z): \quad(m=1, \ldots 4)$, e.g.

$\left.\operatorname{Res} f(z, 1)\right|_{z=0}=2 \gamma-1$

For the function $f(z, m)=\Gamma(z-m) \ldots \Gamma(z-1) \Gamma(z)$

Table $\left[\prod_{n=0}^{m} \Gamma(z-n) / / t F,\{m, 1,4\}\right] / / C F$

$\Gamma(z-1) \Gamma(z)$

$\Gamma(z-2) \Gamma(z-1) \Gamma(z)$

$\Gamma(z-3) \Gamma(z-2) \Gamma(z-1) \Gamma(z)$

$\Gamma(z-4) \Gamma(z-3) \Gamma(z-2) \Gamma(z-1) \Gamma(z)$

the residue is calculated for $z_{0}=\mathbf{0}$ where $\gamma=$ EulerGamma $=0.577216$ and $\psi^{(2)}(1)=$ PolyGamma $[2,1]=-2.40411$.

$\left(\right.$ residues $\left.=\operatorname{Table}\left[\operatorname{Residue}\left[\prod_{n=0}^{m} \Gamma(z-n),\{z, 0\}\right] / / t F,\{m, 1,4\}\right]\right) / / c F$

residues // N

$2 \gamma-1$

$\frac{1}{8}\left(-17+30 \gamma-18 \gamma^{2}-\pi^{2}\right)$

$\frac{1}{324}\left(607-1209 \gamma+936 \gamma^{2}-288 \gamma^{3}+39 \pi^{2}-36 \gamma \pi^{2}+18 \psi^{(2)}(1)\right)$

$\frac{2824919-6394380 \gamma+5988600 \gamma^{2}-2772000 \gamma^{3}+540000 \gamma^{4}+199620 \pi^{2}-277200 \gamma^{2}+108000 \gamma^{2} \pi^{2}+2088 \pi^{4}+110880 \psi^{(2)}(1)-86400 \gamma \psi^{(2)}(1)}{5971968}$

$\{0.154431,-1.94379,0.932612,0.244299\}$

For $z=\mathbf{0}$ the product of $\Gamma$-functions turn out to have the value ComplexInfinity. as is also shown by the subsequent plot. 
$\{f 2=\Gamma(z-2) \Gamma(z-1) \Gamma(z), f 1=\Gamma(z-1) \Gamma(z), f 0=\Gamma(z)\} ;$

Plot $[\{f 2, f 1, f 0\},\{z,-5,4\}$, PlotRange $\rightarrow\{-4,4\}$, AspectRatio $\rightarrow .25$, Frame $\rightarrow$ True,

PlotStyle $\rightarrow$ \{Red, Orange, Blue $\}$, AxesLabel $\rightarrow\{" z ", " "\}$, Background $\rightarrow$ LightGray,

PlotLabel $\rightarrow$ \{Style[f2, Red, Bold], Style[f1, Orange, Bold], Style[f0, Blue, Bold] $\}$, ImageSize $\rightarrow$ 500]

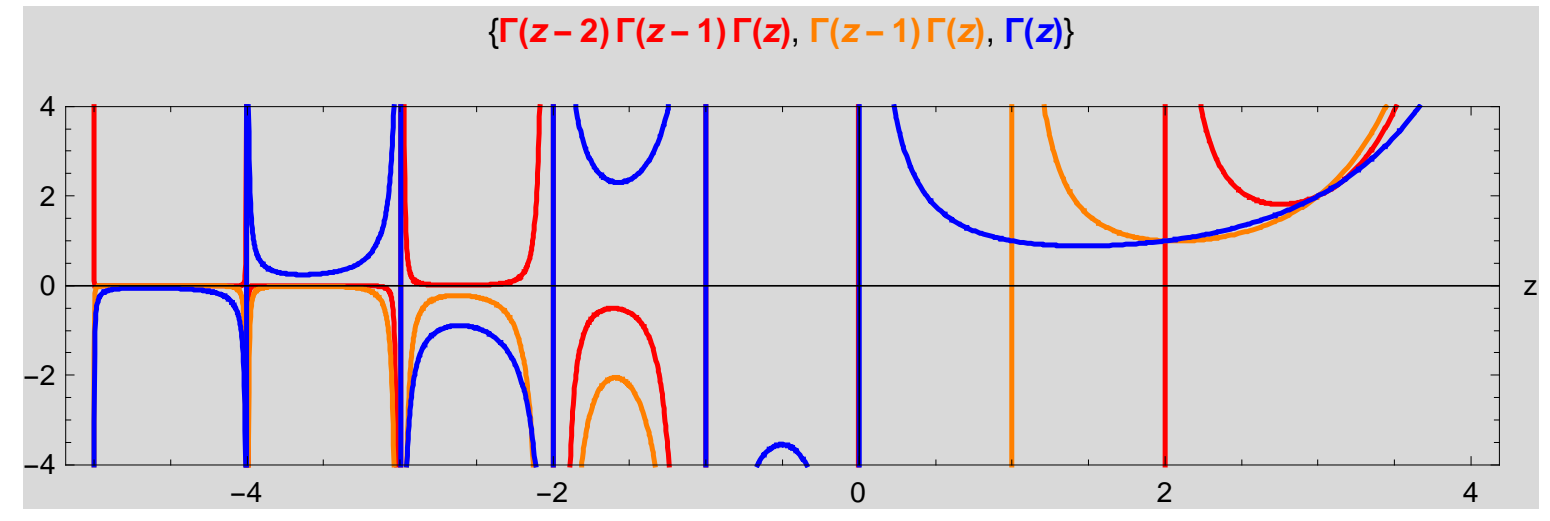

The procedure calculateResidues is not applicable in this case. Instead, it is better to calculate for the products of $\Gamma$-functions a truncated series expansion and extract the coefficient for $z^{\mathbf{1}}$ which is the residuum. (See similar treatment in Example 14)

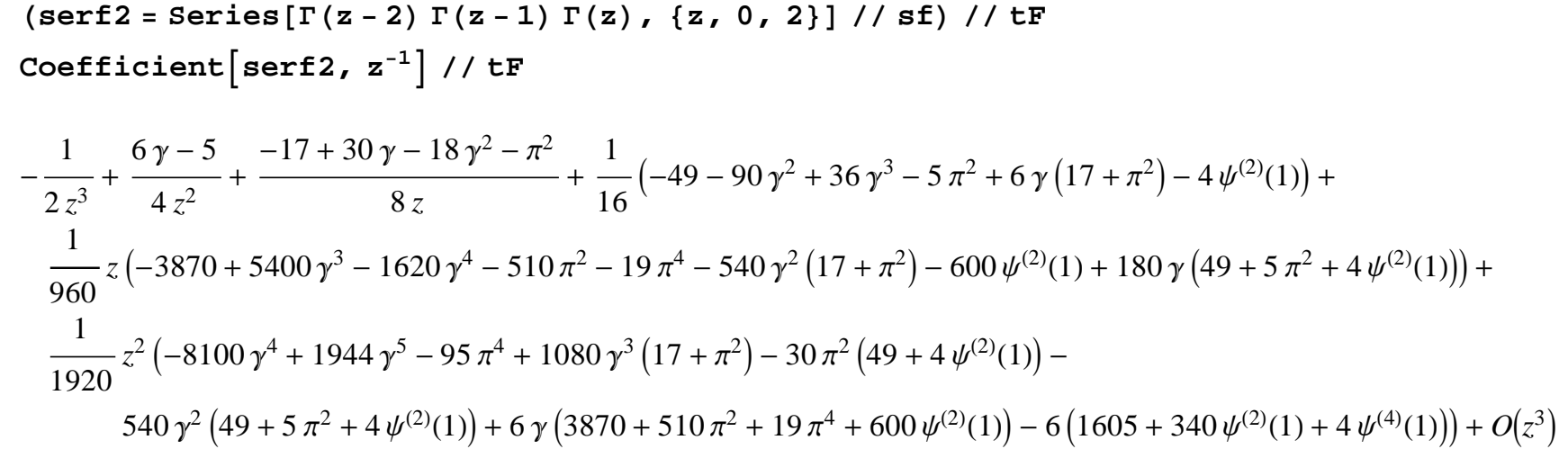


$\frac{1}{8}\left(-17+30 \gamma-18 \gamma^{2}-\pi^{2}\right)$

Example 19 : Residues of $f(z, s)=\Gamma(3+s) \Gamma(s-3) \Gamma\left(1-\frac{1}{2}-s\right) z^{-s}$ :

$$
\left.\operatorname{Res} f(z, s)\right|_{s=3}=-\frac{64 \sqrt{\pi}}{z^{3}}
$$

The integrand of the Meijer $G$-function $G_{11}^{22}(\mathbf{z} \mid \ldots)$ is defined as $f_{\mathbf{2 2 1 1}}(z ; s)=\Gamma(\mathbf{s}+\mathbf{3}) \Gamma(\mathbf{s}-\mathbf{3}) \Gamma\left(\frac{1}{2}-\mathbf{s}\right) z^{-s}$; wanted are the residues for the following sequence of singularities in $s_{\mathbf{0}}=\{\mathbf{3}, \mathbf{2}, \mathbf{1}, \mathbf{0}, \mathbf{- 1}, \ldots,-\mathbf{3}\}$.

For special functions (such as $f_{m n p q}(z, s)$ which essentially consist of $\Gamma$-functions) a modified search strategy must be applied in order to find the singularities. They are determined with the help of the procedure findSingularities ; the subsequent calculation of the corresponding residues is achieved with the modified procedure calculateResidues 4 SpecFct.

\section{? calculateResidues 4 SpecFct}

calculateResidues4SpecFct[f, s, selectPoles_:None, polesRange_:\{\}, onoff_:"Off"] evaluates the residues symbolically. 'f' denotes the special function (e.g. MeijerG-function) $f(s)$ with ' $s$ ' as complex variable $s \in \mathbb{C}$. The singularities of $f(s)$ are determined by calling findSingularities within the range given by 'polesRange' $=\{a, b\}$; the list of lists $\{$ poles, $\mu$,solK $\}$ is returned where 'poles' contains the list of singularities, ' $\mu$ ' is a list containing the multiplicity of every pole and solK $=0$ is set. For special functions the parameter 'selectPoles'= None must be chosen. With an additional global variable $\$ \kappa$ the number of poles can be controlled. Hence, for $\$ k=2$ the index $k$ assumes the values $\{0,1,2\}$ whereas for $\$ K=-2$ there is $k=\{-2,-1,0,1,2\}$. The sum of residues for the singularities selected is calculated and returned. With onoff= "No" printout is suppressed.

Here, the residues are determined for the singularities in the integer values of $\boldsymbol{s}_{\mathbf{0}}$.

$\operatorname{Table}\left[\left\{" s_{0}=", s_{0}, \operatorname{Residue}\left[\Gamma(3+s) \Gamma(-3+s) \Gamma\left(1-\frac{1}{2}-s\right) z^{-s},\left\{s_{,} s_{0}\right\}\right] / / t F\right\},\left\{s_{0}, 3,-3,-1\right\}\right] / / \mathbf{s f} / / \mathbf{c F}$ 
The constants which occur are $\gamma=$ EulerGamma and $\psi^{(0)}\left(\frac{k}{2}\right)=$ PolyGamma $\left[0, \frac{k}{2}\right]$.

Furthermore, the residue theorem has an important practical application to determine the number of zeros $N$ and poles $\boldsymbol{P}$ of a meromorphic function $f(z)$ located within a simple-connected domain $\mathcal{D}$.

\section{Argument principle}

Suppose $\mathcal{D}$ is a simply connected domain and $\gamma$ a simple closed positively oriented contour path in $\mathcal{D}$ such that the meromophic function $\boldsymbol{f}(\boldsymbol{z})=\frac{\left(z-a_{1}\right)^{\alpha_{1}}\left(z-a_{2}\right)^{\alpha_{2}} \ldots\left(z-a_{r}\right)^{\alpha_{r}}}{\left(z-b_{1}\right)^{\beta_{1}}\left(z-b_{2}\right)^{\beta_{2}} \ldots\left(z-b_{s}\right)^{\beta_{s}}} \cdot \boldsymbol{g}(z)$ has no zeros $\boldsymbol{a}_{\boldsymbol{j}}$ or poles $\boldsymbol{b}_{\boldsymbol{k}}$ on $\boldsymbol{\gamma}$. (Apart from the poles $\boldsymbol{b}_{\boldsymbol{k}}$ the function $\boldsymbol{f}(\boldsymbol{z})$ is holomorphic and has by definition no essential singularities. $g(z)$ is analytic and nonzero on and inside $\gamma$.) Then $\frac{1}{2 \pi i} \oint_{\gamma} \frac{f^{\prime}(z)}{f(z)} d z=N-P$ where $\mathbf{N}=\sum_{\mathrm{j}=1}^{\mathrm{r}} \alpha_{\mathrm{j}}$ is the number of zeros and $\mathbf{P}=\sum_{\mathbf{k}=1}^{\mathrm{s}} \boldsymbol{\beta}_{\mathbf{k}}$ is the number of poles of $\boldsymbol{f}(\boldsymbol{z})$ inside the contour $\gamma$ counted according to their multiplicity $\boldsymbol{\mu}$.

For the proof see [7].

In the special case where $\boldsymbol{f}(\boldsymbol{z})$ is a holomorphic function inside $\mathcal{D}$ and possesses only zeros $\mathbf{z}=\boldsymbol{a}_{\boldsymbol{j}}$ but no singularities $\boldsymbol{z}=\boldsymbol{b}_{\boldsymbol{k}}$ then the number of zeros $\boldsymbol{N}$ of $f(z)$ is given by $N=\frac{1}{2 \pi i} \oint_{\gamma} \frac{f^{\prime}(z)}{f(z)} d z$. This case is important for the numerical determination of zeros of a holomorphic function. Particularly for rational functions the location of zeros plays an important role for stability investigations of mechanical and electrical systems. With the help of Mathemat$i c a$ it is possible to calculate the zeros and poles of the meromorphic function $f(z, r, s)=\prod_{j=1}^{r}\left(z-a_{j}\right)^{\alpha_{j}} / \prod_{k=1}^{s}\left(z-b_{k}\right)^{\beta_{k}}$.

Clear $[f, g]$

$\mathbf{f}\left[\mathbf{z}_{-}, \mathbf{r}_{-}, \mathbf{s}_{-}\right]:=\left(\prod_{j=1}^{\mathbf{r}}(\mathbf{z}-\mathbf{a}[j])^{\alpha[j]}\right) /\left(\prod_{k=1}^{s}(\mathbf{z}-\mathbf{b}[\mathbf{k}])^{\beta[k]}\right)$

$g=\partial_{z} \log [f[z, r, s]] / / \mathbf{s f}$ 
$\frac{\partial_{z} \prod_{j=1}^{r}(z-a[j])^{\alpha[j]}}{\prod_{j=1}^{r}(z-a[j])^{\alpha[j]}}-\frac{\partial_{z} \prod_{k=1}^{s}(z-b[k])^{\beta[k]}}{\prod_{k=1}^{s=1}(z-b[k])^{\beta[k]}}$

In the practical case of $\mathbf{2}$ zeros $\left\{\boldsymbol{a}_{1}, \boldsymbol{a}_{2}\right\}$ with multiplicities $\left\{\alpha_{1}, \alpha_{2}\right\}$ and $\mathbf{3}$ poles $\left\{\boldsymbol{b}_{1}, \boldsymbol{b}_{2}, \boldsymbol{b}_{3}\right\}$ with multiplicities $\left\{\boldsymbol{\beta}_{\mathbf{1}}, \boldsymbol{\beta}_{\mathbf{2}}, \boldsymbol{\beta}_{\mathbf{3}}\right\}$ the logarithmic derivative for the meromorphic function $f(z, 2,3)$ is given by

$g 23=g / . \quad\{r \rightarrow 2, s \rightarrow 3\} / / f s$

$\frac{\alpha[1]}{z-a[1]}+\frac{\alpha[2]}{z-a[2]}-\frac{\beta[1]}{z-b[1]}-\frac{\beta[2]}{z-b[2]}-\frac{\beta[3]}{z-b[3]}$

Furthermore, for zeros $\boldsymbol{a}_{\mathbf{1}}, \boldsymbol{a}_{\mathbf{2}}$ and poles $\boldsymbol{b}_{\mathbf{1}}, \boldsymbol{b}_{\mathbf{2}}, \boldsymbol{b}_{\mathbf{3}}$ the following values are assumed. :

$\{a[1], a[2], b[1], b[2], b[3]\} \in$ Reals;

Setoptions [ Integrate, Principalvalue $\rightarrow$ True,

Assumptions $\rightarrow\{0<a[1]<a[2]<b[1]<b[2]<b[3]<1\}]$

zerosAndPoles $=\left\{a[1] \rightarrow \frac{1}{3^{3}}, a[2] \rightarrow \frac{1}{3^{2}}, b[1] \rightarrow \frac{1}{2^{3}}, b[2] \rightarrow \frac{1}{2^{2}}, b[3] \rightarrow \frac{1}{2}\right\} ;$

In order to evaluate the contour integral $\oint_{\gamma} \frac{f^{\prime}(z)}{f(z)} \boldsymbol{d z}$ with 2 zeros and 3 poles located in $[-\mathbf{1},+\mathbf{1}]$ the closed contour $\gamma$ encircles this intervall counterclockwise. The path from $\mathbf{+ 1} \rightarrow-\mathbf{- 1}$ is slightly above the real axis and the path from $\mathbf{- 1} \rightarrow \mathbf{+ 1}$ is slightly below the real axis. Thus

$\epsilon=10^{-20}$

$\mathcal{J}=\left(\frac{1}{2 \pi \dot{i}}\left(\int_{+1+\dot{i} \epsilon}^{1+\dot{i} \epsilon} g 23 d z+\int_{-1-\dot{i} \epsilon}^{+1-\dot{i} \epsilon}(g 23) d z\right) /\right.$ zerosAndPoles $) / / N / /$ sf // Rationalize

$\alpha[1]+\alpha[2]-\beta[1]-\beta[2]-\beta[3]$

or comparing the result above with the direct calculation of $\mathcal{N}-\mathcal{P}$ 
$\mathcal{J}==\sum_{j=1}^{2} \alpha[j]-\sum_{k=1}^{3} \beta[k]$

True

Obviously, the numerical value of the contour integral $\mathcal{J}$ is equal to the difference of the numbers of zeros $N=\alpha_{1}+\alpha_{2}$ and the numbers of poles $\boldsymbol{P}=\beta_{1}+\beta_{2}+\beta_{3}$, which are counted according to their multiplicities $\left\{\alpha_{1}, \alpha_{2}\right\}$ and $\left\{\beta_{1}, \beta_{2}, \beta_{3}\right\}$.

Example 20 : $\quad \frac{1}{2 \pi i} \oint_{C} \frac{f^{\prime}(z)}{f(z)} d z$ with $f(z)=\left(z^{2}+i z-\frac{1}{2}\right) \Longrightarrow \sum_{i=1}^{2} \operatorname{Res} \frac{\overline{\tilde{D}}+2 z}{-\frac{1}{2}+\bar{l} z+z^{2}} \mid z=( \pm 1-\bar{l}) / 2=2$

Given is the meromorphic function $f(z)=\left(z^{2}+i z-\frac{1}{2}\right)$. The zeros $\mathbf{z}_{1,2}=( \pm \mathbf{1}-\boldsymbol{i}) / \mathbf{2}$ are obtained solving the equation $f(z)=\mathbf{0}$ w.r.t. the variable $z$.

$\mathrm{z}=. ; \mathrm{z} 0=. ; \mathrm{f}:=\left(\mathrm{z}^{2}+\dot{\mathrm{I}} \mathrm{z}-\frac{1}{2}\right)$

$z 0=($ Solve $[f==0, z] / /$ Flatten $) /. \operatorname{Rule}\left[a_{-}, b_{-}\right] \rightarrow b$

$\left\{-\frac{1}{2}-\frac{\dot{1}}{2}, \frac{1}{2}-\frac{\dot{1}}{2}\right\}$

According to the argument principle given above the number $\mathcal{N}$ of zeros $\mathbf{z 0}_{j}$ of the function $f(z)$ are obtained from the contour integral $\mathcal{N}=\frac{1}{2 \pi i} \oint_{\gamma} g(z) d z=\left.\sum_{j} \operatorname{Res} \frac{f^{\prime}(z)}{f(z)}\right|_{z=u_{j}}$ where $g(\mathbf{z})=\frac{f^{\prime}(z)}{f(z)}=\frac{i+2 \mathrm{z}}{\left(\mathrm{z}^{2}+i \mathrm{z}-\frac{1}{2}\right)}$

$\mathcal{N}=\sum_{i=1}^{2} \operatorname{Residue}\left[g=\partial_{z} \log [f],\{z, z 0 \llbracket i \rrbracket\}\right]$

2

The same result is obtained with ContourIntegral multiplied by the factor $1 /(2 \pi i)$.

ContourIntegral[g, z, All, \{\}, No"] / ( $2 \pi \dot{i})$ 
Contour integral $\mathcal{J}=\oint \frac{\dot{\mathbb{1}}+2 \mathrm{z}}{-\frac{1}{2}+\dot{1} z+z^{2}} \mathrm{~d} z=4 \dot{i} \pi$

\section{- Contour Integration}

\section{- Motivation for Symbolic Contour Integration}

Contour integration is a method in complex analysis for the calculation of certain integrals along a path $\gamma$ in the complex plane $\mathbb{C}$; this method is closely related to the calculus of residues as shown before. One use of contour integrals is the evaluation of integrals along the real axis that are not easily determined by using only methods with real variables. The main applications of contour integration are :

- integration of a complex- valued function $f(z)$ along a curve $\boldsymbol{\gamma}$ in $\mathbb{C}$,

- application of Cauchy's integral theorem,

- application of residue theorem.

- definition of special functions in terms of contour integral representation.

See, for example, the definition of the $\Gamma$-function in terms of Hankel's contour integral as found on The Wolfram Functions Site [13]

$\Gamma(z)=\frac{1}{e^{2 \pi i z}-1} \int_{\mathcal{L}} e^{-t} \boldsymbol{t}^{z-1} d t$. The path of integration $\mathcal{L}$ starts at $\infty+\boldsymbol{i} \mathbf{0}$ above the real axis, goes to $\rho+\boldsymbol{i} \mathbf{0}$, encircles the origin in counter-clockwise direction with radius $\boldsymbol{\rho}$ to the point $\boldsymbol{\rho}-\boldsymbol{i} \mathbf{0}$ below the real axis, and returns to the point $\infty-\boldsymbol{i} \mathbf{0}$.

$$
\Gamma\left(z_{-}\right):=\frac{1}{e^{2 \pi \dot{i} z_{-}}} \text {ContourIntegrate }\left[e^{-t} t^{z-1},\{t, \mathcal{L}\}\right]
$$

A Mathematica procedure ContourIntegrate $[\mathbf{f}[\mathbf{z}, \mathbf{t}],\{\mathbf{t}, \mathcal{L}\}]$ is suggested for the (symbolic) calculation of the contour integrals. However, this essential procedure is not yet implemented in Mathematica nor available elsewhere which is astonishing. There are several reason why the implementation has not been done. 
According to private communications with M. Trott / WRI (2010) who together with Oleg Marichev essentially contributed to Function Site there are various resons why the implementation of ContourIntegrate has not been done yet.

Thus, this was - apart from other reasons - motivation for the author implementing such a procedure by himself which covers many non-trivial contour integrals but does not claim to treat all possible cases.

\section{? Contourintegral}

ContourIntegral[f, z, selectPoles_All, polesRange_\{\}, onoff_"On"] evaluates contour integrals symbolically in the complex plane $\mathbb{C}$ by means of the residues for the poles selected. ' $f$ ' denotes the integrand $f(z)$ of the contour integral where ' $z$ ' is the integration variable $z \in \mathbb{C}$. If the parameter 'selectPoles' is given as $\{\mathrm{i}, \mathrm{j}, \ldots\}$ then a subset of poles $\mathrm{z}_{\mathrm{i}}, \mathrm{z}_{\mathrm{j}}, \ldots$ is considered only for residues; default value for 'selectPoles' is 'All' considering all poles for the calculation of residue. The sum of residues is evaluated with the procedure calculateResidues[...]. However, if the parameter 'selectPoles' is 'SpecFct' then $f(z)$ is assumed being a special function, for example the integrand $f_{m n p q}(z, s)$ of the Meijer G-function. The parameter 'polesRange' $=\left\{s_{\min }, s_{\max }\right\}$ confines the search range in which findSingularities [...] determines the singularities. Procedure calculateResidues4SpecFct[...] evaluates the corresponding residues. Finally, the sum of residues will be multiplied by $2 \pi i$ and returned in the variable $\mathcal{J}$ as value for the contour integral. With "No" intermediate printout is complete suppressed.

In this context it is near at hand to introduce a special notation for contour integrals as was done before.

$$
\oint_{\text {selectPoles }_{-} \text {polesRange }, \text { onoff }} \mathbf{f}_{-} \mathrm{dlz_{- }} \Leftrightarrow \text { ContourIntegral }\left[\mathbf{f}_{-}, \mathbf{z}_{-}, \text {selectPoles_, polesRange }, \text { onoff }\right]
$$

As an example the complex function $f(z)=\frac{1}{\left(z^{3}+1\right)^{2}}$ is considered. $f(z)$ has three double poles at $z_{1}=-\mathbf{1}$ and $z_{2,3}=(\mathbf{1} \mp i \sqrt{\mathbf{3}}) / \mathbf{2}$, Taking into account only the poles $z_{2,3}$ the contour integral yields

$$
\text { ContourIntegral }\left[\frac{1}{\left(z^{3}+1\right)^{2}}, z,\{2,3\},\{\}, \text { "On" }\right]
$$


The same result is obtained with the special notation with the symbol $\oint \ldots$

$$
\mathcal{J}=\oint_{\{2,3\},\{\}, \text { No" }} \frac{1}{\left(z^{3}+1\right)^{2}} d \mathbf{z}
$$

Contour integral $\mathcal{J}=\oint \frac{1}{\left(1+z^{3}\right)^{2}} d z=-\frac{4 \dot{i} \pi}{9}$

$-\frac{4 \dot{i} \pi}{9}$

The examples for contour integration are classified according to the following groups of functions :

- (1) Rational polynomials $f(z)=\frac{p(z)}{q(z)}$

- (2) Products of rational polynomials and trigonometric functions $f(z)=\frac{p(z)}{q(z)}\left\{\begin{array}{l}\cos (\alpha z) \\ \sin (\alpha z)\end{array}\right.$

- (3) Products of rational polynomials and hyperbolic functions $f(z)=\frac{p(z)}{q(z)}\left\{\begin{array}{l}\cosh (\alpha z) \\ \sinh (\alpha z)\end{array}\right.$

- (4) Rational functions of trigono./hyperbol. functions $F(\sin (\theta), \cos (\theta)), F(\sinh (\theta), \cosh (\theta))$

Further examples obtained by transformation of indefinite integrals into contour integrals by appling a change of integration variables and contour integrals with branch cuts will be investigated in Part 2 of a subsequent paper.
- Rational polynomials $f(z)=\frac{p(z)}{q(z)}$
(Examples 1-21)

In order to calculate improper integrals $\int_{-\infty}^{+\infty} \frac{p(x)}{q(x)} d x$ with integrands being rationalfunctions ( that consist of complex polynomials $p(z), q(z)$ where $\mathbf{q}(\mathbf{x})$ $\neq \mathbf{0}$ for each real value of $\mathbf{x} \in \mathbb{R}$ ) it is assumed that the (extended) contour path $\gamma$ is closed in the complex half-plane $\mathbf{H}_{ \pm}$. It is supposed that the contribution of the integral over the semi-circle $\boldsymbol{C}_{\boldsymbol{R}}$ with radius $\boldsymbol{R} \rightarrow \infty$ vanishes. Hence 


$$
\mathcal{J}=\int_{-\infty}^{+\infty} \frac{p(x)}{q(x)} d x=\oint_{\gamma} \frac{p(z)}{q(z)} d z= \pm\left. 2 \pi i \sum_{j=1}^{k} \operatorname{Res} \frac{p(z)}{q(z)}\right|_{z=z_{j}}
$$

If $\boldsymbol{q}(z)$ has at least one zero (in general, $\left\{z_{1}, z_{2}, \ldots, z_{k}\right\}$ denote the zeros of $\boldsymbol{q}(z)$ in the upper half-plane $\mathbb{H}_{+}$) then the contour path $\gamma$ is closed in the upper half-plane. The sign depends on the orientation of the contour $\gamma$ : if the path orientation is in the mathematical positive sense, i.e. anti-clockwise, then the positive sign hold; if, however, the orientation is in the mathematical negative sense, i.e. clock-wise, then the negative sign holds for the integral.

\section{Order of $\mathrm{q}(\mathrm{z}): n=1$}

Example 1: $\oint_{\gamma} \frac{f(z)}{z} d z=2 \pi$ if $[0]$

Example 2: $\oint_{\gamma}\left(\frac{1}{z+1}+\frac{1}{z-1}+\frac{1}{z+i}-\frac{1}{z-i}\right) d z=8 \pi i$

The integrand consits of a sum of polynomials $\boldsymbol{q}_{\boldsymbol{i}}(\mathbf{z})$ with simple poles $\{\mathbf{1}, \mathbf{- 1}, \boldsymbol{i}, \boldsymbol{i} \boldsymbol{i}\}$. Because a single function is expected as the first argument the terms must be combined with Together.

$\zeta=\{1,-1, \dot{\mathbf{i}},-\dot{\mathbf{i}}\} ; \mathbf{f}\left[\mathbf{z}_{-}, \zeta\right]=\sum_{\mathrm{k}=1}^{4} \frac{1}{(\mathbf{z}+\zeta \llbracket \mathrm{k} \rrbracket)} / /$ Together

ContourIntegral $[f[z, \zeta], z, A l 1,\{\}, "$ Off" $]$;

$\frac{4 z^{3}}{(-1+z)(-\dot{i}+z)(\dot{1}+z)(1+z)}$

$f(z)=\frac{4 z^{3}}{(z-1)(z-\mathbb{i})(z+\mathbb{i})(z+1)}$ has poles with multiplicity
$\quad\left\{\left\{z_{i}, \mu_{i}\right\}, \ldots\right\}=\{\{-1,1\},\{-\dot{i}, 1\},\{1,1\},\{\dot{1}, 1\}\} \Leftrightarrow$ residues $=\{1,1,1,1\}$ 
ContourIntegration_P1.nb

Residues $\left.\sum_{i} \operatorname{Res}(f(z))\right|_{z=z_{i}}=4$ for pole $(s) i=A l l$

Contour integral $\mathcal{J}=\oint \frac{4 z^{3}}{(-1+z)(-\dot{i}+z)(\dot{1}+z)(1+z)} d z=8 \dot{i} \pi$

Order of $q(\mathrm{z}): n=2$

Example 3 : $\oint \frac{1}{1+z^{2}} d z=\pi$

Example $4: \oint_{\gamma} \frac{2 z-3}{z(z-1)} d x=4 i \pi$

Example 5: $\oint_{V} \frac{z^{2}+1}{z(z-1)} d z=2 i \pi$

Example 6: $\oint_{\gamma} \frac{e^{z}}{z(z+2)} d z=i \pi\left(1-e^{-2}\right)$

The function $f(z)=\frac{\Theta^{2}}{z(z+2)}$ has two single poles at $z_{1}=-\mathbf{2}$ and another one in $z_{2}=\mathbf{0}$.

ContourIntegral $\left[\frac{e^{z}}{z(z+2)}, \mathbf{z}, A l l,\{\}\right.$, "Off" $]$;

$f(z)=\frac{\mathbb{e}^{z}}{z(z+2)}$ has poles with multiplicity

$\left\{\left\{z_{i}, \mu_{i}\right\}, \ldots\right\}=\{\{-2,1\},\{0,1\}\} \quad \Leftrightarrow \quad$ residues $=\left\{-\frac{1}{2 \mathbb{e}^{2}}, \frac{1}{2}\right\}$

145 
Residues $\left.\sum_{i} \operatorname{Res}(f(z))\right|_{z=z_{i}}=\frac{1}{2}-\frac{1}{2 e^{2}}$ for $\operatorname{pole}(s) i=A l l$

Contour integral $\mathcal{J}=\oint \frac{\mathbb{e}^{z}}{\mathrm{z}(2+\mathrm{z})} \mathrm{dz}=\mathbf{i}\left(\pi-\frac{\pi}{\mathbb{e}^{2}}\right)$

Example 7: $\oint_{V} \frac{4 \mathrm{z}-3}{\mathrm{z}(\mathrm{z}-2)} d \mathrm{z}=8 i \pi$

Example 8: $\oint_{\gamma} \frac{1}{\left(z^{2}-4 z+3\right)} d z=i \pi$

Example 9: $\oint_{\gamma} \frac{4 z^{4}-3 z^{2}+5}{(z-1)(z+5)} d z=2 \pi i$

Order of $q(z): n=3$

Example 10 : $\oint_{\gamma} \frac{1}{\left(z^{2}-4 z+4\right)(z-6-i)} d z=\frac{2 \pi}{289}(8+15 i)$

Order of $q(z): n=4$

Example 11: $\oint_{\gamma} \frac{2 z^{2}+z+1}{\left(z^{2}+1\right)(z-2 i)^{2}} d z=0$ ( respectively $\frac{\pi}{9}(1+i)$ for $\left.z_{2,3} \in \mathbb{H}_{+}\right)$

The rational $f(z)=\frac{2 z^{2}+z+1}{\left(z^{2}+1\right)(z-2 i)^{2}}$ has two single poles at $z_{1,2}=\mp i$ and a double pole in $z_{3}=2 i$. If all poles are taken into account the value of the contour integral is 0 . Separately, the residues are obtained as

Pole $z_{1}=+i$ has with $g(z)=(z-i) f(z)=\frac{2 z^{2}+z+1}{(z+i)(z-2 i)^{2}}$ the residue $\left.\operatorname{Res} f(z)\right|_{z=+i}=g(i)=-\frac{1+i}{2}$;

Pole $z_{2}=-i$ has with $g(z)=(z+i) f(z)=\frac{2 z^{2}+z+1}{(z-i)(z-2 i)^{2}}$ the residue $\left.\operatorname{Res} f(z)\right|_{z=-i}=g(-i)=-\frac{1-i}{18}$; 
Double pole $z_{3}=2 i$ has with $g(z)=(z-2 i) f(z)=\frac{2 z^{2}+z+1}{\left(z^{2}+1\right)}$ the residue $\left.\operatorname{Res} f(z)\right|_{z=2 i}=g^{\prime}(2 i)=-\frac{5+4 i}{9}$;

thus the sum of residues is $: \oint_{C} f(z) d z=\left.2 \pi i \sum_{j=1}^{3} \operatorname{Res} f(z)\right|_{z=z_{j}}=2 \pi i\left(-\frac{1+i}{2}-\frac{1-i}{18}-\frac{5+4 i}{9}\right)=0$.

ContourIntegral $\left[\frac{2 z^{2}+z+1}{\left(z^{2}+1\right)(z-2 \text { i })^{2}}, z, A l l,\{\}, " O f f "\right]$;

$f(z)=\frac{2 z^{2}+z+1}{(z-2 \dot{1})^{2}\left(z^{2}+1\right)}$ has poles with multiplicity

$\left\{\left\{\mathrm{z}_{i}, \mu_{i}\right\}, \ldots\right\}=\{\{-\dot{\mathbb{1}}, 1\},\{\dot{\mathbb{1}}, 1\},\{2 \dot{\mathbb{1}}, 2\}\} \Leftrightarrow$ residues $=\left\{-\frac{1}{18}+\frac{\dot{i}}{18},-\frac{1}{2}+-\frac{\dot{i}}{2}, \frac{5}{9}+\frac{4 \dot{i}}{9}\right\}$

$\left.\operatorname{Residues} \sum_{i} \operatorname{Res}(f(z))\right|_{z=z_{i}}=0$ for pole $(s) \quad i=\operatorname{All}$

Contour integral $\mathcal{J}=\oint \frac{1+z+2 z^{2}}{(-2 \dot{i}+z)^{2}\left(1+z^{2}\right)} d z=0$

However, if only poles $\boldsymbol{z}_{\mathbf{2}, \mathbf{3}} \in \mathbf{H}_{+}$the value of the contour integral is

ContourIntegral $\left[\frac{2 z^{2}+z+1}{\left(z^{2}+1\right)(z-2 \dot{i})^{2}}, z,\{2,3\},\{\}, " N o "\right]$;

Contour integral $\mathcal{J}=\oint \frac{1+z+2 z^{2}}{(-2 \dot{i}+z)^{2}\left(1+z^{2}\right)} d z=\left(\frac{1}{9}+\frac{\dot{i}}{9}\right) \pi$ 
Example 12 : $\oint_{\gamma} \frac{1}{1+z^{2}+z^{4}} d z=\frac{\pi}{\sqrt{3}}$

Example 13: $\frac{1}{2} \oint_{\gamma} \frac{z^{2}}{\left(z^{2}+1\right)\left(z^{2}+9\right)} d z=\frac{\pi}{8}$

Example $14: \oint_{\gamma} \frac{1}{z^{3}(1+5)} d z=-\frac{2 \pi}{125} i$

Order of $q(z): n=5$

Example 15: $\oint_{\gamma} \frac{e^{2 z}}{(1-z)^{5}} d z=-\frac{4}{3} \tilde{\bar{D}} \pi \Theta^{2}$

Example $16: \oint_{\gamma} \frac{f(z)}{\left(z^{5}-1\right)} d z=\frac{1}{10} \pi\left(4 \tilde{u} \tilde{f}[1]+(\sqrt{10-2 \sqrt{5}}-\tilde{i}(1+\sqrt{5}))+\left[\left[-(-1)^{1 / 5}\right]-\tilde{i} \tilde{t}\left[(-1)^{2 / 5}\right]+\tilde{i} \sqrt{5} \mathrm{f}\left[(-1)^{2 / 5}\right]-\right.\right.$

$$
\sqrt{2(5+\sqrt{5})} \mathrm{f}\left[(-1)^{2 / 5}\right]-\tilde{\tilde{t}} \mathrm{f}\left[-(-1)^{3 / 5}\right]+\tilde{L} \sqrt{5} \mathrm{f}\left[-(-1)^{3 / 5}\right]+\sqrt{2(5+\sqrt{5})} \mathrm{f}\left[-(-1)^{3 / 5}\right]-\tilde{I} \mathrm{f}\left[(-1)^{4 / 5}\right]-\tilde{I} \sqrt{5} \mathrm{f}\left[(-1)^{4 / 5}\right]-\sqrt{10-2 \sqrt{5}} \mathrm{f}\left[(-1)^{4 / 5}\right]
$$

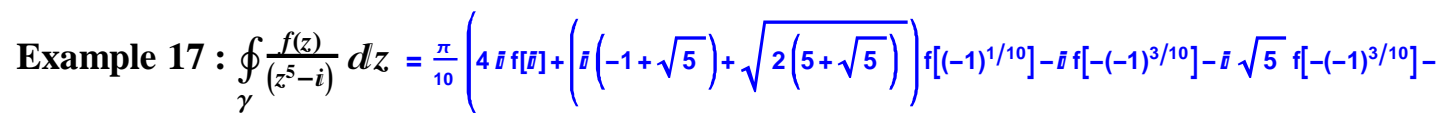

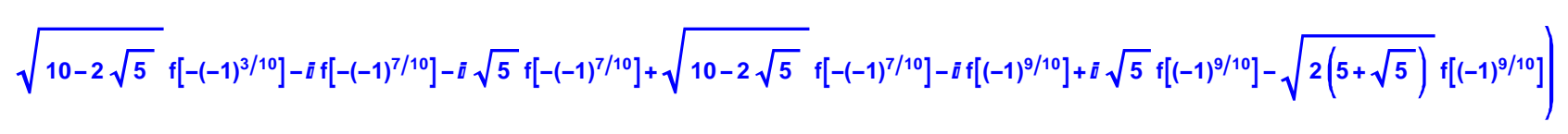

Here, the poles of $\frac{f(z)}{\left(z^{5}-i\right)}$ are the roots of $\sqrt[5]{i}$

$z=$;

poles $=\left(\right.$ Solve $\left[\mathbf{z}^{5}=\dot{\mathbf{i}}, \mathbf{z}\right] / /$ ce $/ /$ Flatten $) / .\{$ Rule $\rightarrow$ Set $\}$

poles // fs 
$\left\{\dot{\mathbf{i}}, \sqrt{\frac{5}{8}+\frac{\sqrt{5}}{8}}+\dot{\mathbb{i}}\left(-\frac{1}{4}+\frac{\sqrt{5}}{4}\right), \dot{\mathbf{i}}\left(-\frac{1}{4}-\frac{\sqrt{5}}{4}\right)-\sqrt{\frac{5}{8}-\frac{\sqrt{5}}{8}}, \dot{\mathbf{i}}\left(-\frac{1}{4}-\frac{\sqrt{5}}{4}\right)+\sqrt{\frac{5}{8}-\frac{\sqrt{5}}{8}},-\sqrt{\frac{5}{8}}+\frac{\sqrt{5}}{8}+\dot{i}\left(-\frac{1}{4}+\frac{\sqrt{5}}{4}\right)\right\}$

$\left\{\dot{1},(-1)^{1 / 10},-(-1)^{3 / 10},-(-1)^{7 / 10},(-1)^{9 / 10}\right\}$

Remove [f]; $\mathbf{z}=$. ;

ContourIntegral $\left[\frac{\mathbf{f}[\mathbf{z}]}{\mathbf{z}^{5}-\dot{\mathbf{i}}}, \mathbf{z}, \mathrm{All},\{\}\right.$, No"] ;

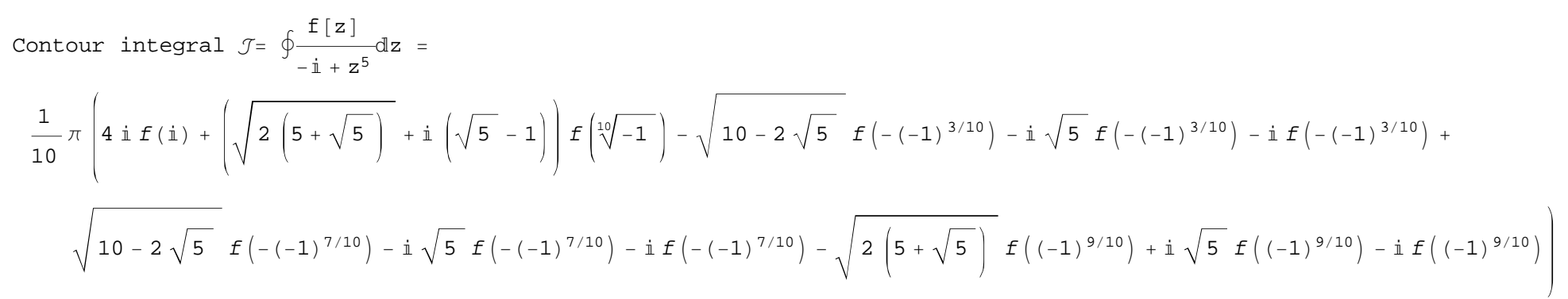

\section{Order of $q(z): n>5$}

Example 18: $\oint_{\gamma} \frac{2 z^{2}+17}{(z-1)^{3}(z+1)^{3}} d z=-\frac{49}{8} \overline{0} \pi$

Example 19: $\oint_{\gamma} \frac{z^{2}}{\left(z^{2}+1\right)\left(z^{2}+4\right)^{2}} d z=\frac{\pi}{36}$

Example 20: $\oint_{\gamma} \frac{1}{\left(1-z^{2}\right)^{2}\left(1+z^{2}\right)^{3}} d z=\frac{13 \pi}{32}$

$f(z)=\frac{1}{\left(1-z^{2}\right)^{2}\left(1+z^{2}\right)^{3}}$ has two double poles in $z_{1,3}=\mp 1$ and two triple poles in $z_{2,4}=\mp i$. If the contour $\gamma$ is a circle around $z_{4}=+i$ with radius $R=\frac{1+\sqrt{5}}{2}=1.62 \ldots$ then only the poles $z_{1}=-1$ and $z_{3}=+1$ are located inside the contour because $\left|z_{4}-z_{1,3}\right|=\sqrt{2}$. 
ContourIntegral $\left[\frac{1}{\left(1-\mathbf{z}^{2}\right)^{2}\left(1+\mathbf{z}^{2}\right)^{3}}, \mathbf{z},\{1,3,4\},\{\}\right.$, "Off" $]$;

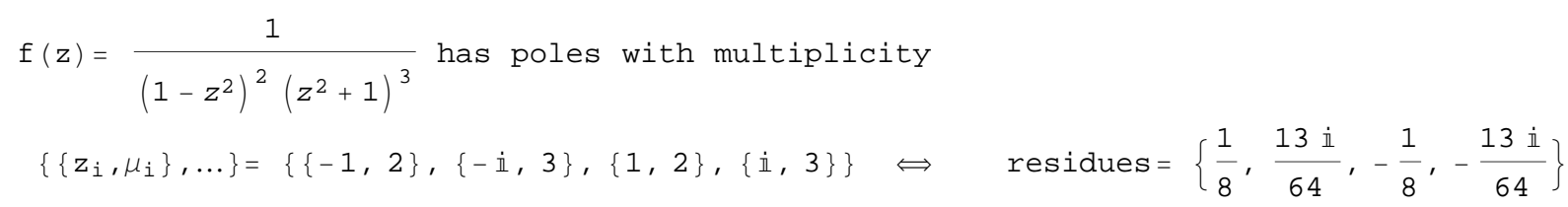

Obviously the residues for $z_{1}$ and $z_{3}$ cancel each other; therefore the same result is obtained if only $z_{4}=+\boldsymbol{i}$ is taken into account.

ContourIntegral $\left[\frac{1}{\left(1-z^{2}\right)^{2}\left(1+z^{2}\right)^{3}}, z,\{4\},\{\}\right.$, No" $]$;

Contour integral $\mathcal{J}=\oint \frac{1}{\left(1-z^{2}\right)^{2}\left(1+z^{2}\right)^{3}} d z=\frac{13 \pi}{32}$

Example 21: $\int_{-\infty}^{+\infty} \frac{(n-1) !}{w(w-z)^{n}} d w=2 \pi i\left(-\frac{1}{\mathrm{z}}\right)^{\mathrm{n}}(1)_{\mathrm{n}-1} \quad(n=1,2, \ldots)$

With series of functions $\left\{f_{1}=\frac{1}{w(w-z)}, f_{2}=\frac{1 !}{w(w-z)^{2}}, f_{3}=\frac{2 !}{w(w-z)^{3}}, \ldots, f_{n}=\frac{(n-1) !}{w(w-z)^{n}}\right\} \quad(n=1,2, \ldots)$ generated from $f_{1}(w, z)$ by application of successive derivatives $\partial_{\{z, \mathrm{n}\}} f_{1}(w, z)$ one obtains 
$\mathbf{z}=. ; \mathbf{w}=$.

$f[1]=\frac{1}{w(w-z)} ; f\left[n_{-}\right]:=\partial_{\{z, n-1\}} f[1] ; \operatorname{Table}[f[n],\{n, 1,5\}]$

$\left\{\frac{1}{w(w-z)}, \frac{1}{w(w-z)^{2}}, \frac{2}{w(w-z)^{3}}, \frac{6}{w(w-z)^{4}}, \frac{24}{w(w-z)^{5}}\right\}$

Table $[g[n][z]=$ ContourIntegral $[f[n], w,\{1\},\{\}$, No"], $\{n, 1,5\}]$

Contour integral $\mathcal{J}=\oint \frac{1}{\mathrm{w}(\mathrm{w}-\mathrm{z})} d \mathrm{w}=-\frac{2 \dot{\mathbf{i}} \pi}{\mathrm{z}}$

Contour integral $\mathcal{J}=\oint \frac{1}{\mathrm{w}(\mathrm{w}-\mathrm{z})^{2}} \mathrm{dw}=\frac{2 \dot{\mathrm{i}} \pi}{\mathrm{z}^{2}}$

Contour integral $\mathcal{J}=\oint \frac{2}{\mathrm{w}(\mathrm{w}-\mathrm{z})^{3}} \mathrm{~d} \mathrm{w}=-\frac{4 \dot{i} \pi}{\mathrm{z}^{3}}$

Contour integral $\mathcal{J}=\oint \frac{6}{\mathrm{w}(\mathrm{w}-\mathrm{z})^{4}} \mathrm{~d} \mathrm{w}=\frac{12 \text { i } \pi}{\mathrm{z}^{4}}$

Contour integral $\mathcal{J}=\oint \frac{24}{\mathrm{w}(\mathrm{w}-\mathrm{z})^{5}} \mathrm{dw}=-\frac{48 \text { i } \pi}{z^{5}}$

$\left\{-\frac{2 \dot{\mathbf{i}} \pi}{z}, \frac{2 \dot{\mathbf{i}} \pi}{z^{2}},-\frac{4 \dot{\mathbf{i}} \pi}{z^{3}}, \frac{12 \dot{\mathbf{i}} \pi}{z^{4}},-\frac{48 \dot{\mathbf{i}} \pi}{z^{5}}\right\}$

This sequence can be casted into a closed form with help of the Pochhammer symbol $(\mathbf{1})_{m-1} \equiv$ Pochhammer $[1,-1+m]$ 
Table $[g[n][z],\{n, 1,5\}] / /$ FindSequenceFunction[\#][n]\&

$$
2 \text { i } \pi\left(-\frac{1}{\mathrm{z}}\right)^{\mathrm{n}} \text { Pochhammer }[1,-1+\mathrm{n}]
$$

In conclusion : according to Cauchy's integral theorem : $g(z)=\frac{1}{2 \pi i} \oint_{\gamma} \frac{f(w)}{w-z} d w$ and $\partial_{\{z, m\}} g(z)=\oint_{\gamma} \partial_{\{z, m\}}\left(\frac{f(w)}{w-z}\right) d w$ it is demonstrated that the $m$ th derivative of the antiderivative $g(z)$ is equal to the corresponding $m$-th derivative of $f(z)$ which is the integrand of the contour integral.

$\mathrm{m}=15$;

$\partial_{\{z, m\}} g[1][z]===$ ContourIntegral $\left[\partial_{\{z, m\}} f[1], w,\{1\},\{\}, " N o "\right]$

Contour integral $\mathcal{J}=\oint \frac{1307674368000}{\mathrm{w}(\mathrm{w}-\mathrm{z})^{16}} \mathrm{dw}=\frac{2615348736000 \text { i } \pi}{z^{16}}$

True

- Product of rational and trigonometric functions $f(z)=\frac{p(z)}{q(z)} \cdot\left\{\begin{array}{l}\cos (\alpha z) \\ \sin (\alpha z)\end{array}\right.$

Moreover, even for products of rational and trigonometric functions the residue theorem and contour integration can be applied likewise.

\section{$\mathcal{J}=\int_{-\infty}^{+\infty} \frac{p(x)}{q(x)} \cdot\left\{\begin{array}{c}\cos (\alpha x) \\ \sin (\alpha x)\end{array} d x \quad\right.$ or $\quad \mathcal{J}=\int_{-\infty}^{+\infty} \frac{p(x)}{q(x)} e^{i \alpha x} d x$}

Example 22 : $\int_{0}^{+\infty} \frac{\cos (\alpha z)}{1+z^{2}} d z=\frac{e^{-\alpha} \pi}{2} \quad(\alpha>0)$

Example 23: $\oint_{\gamma} \frac{\cos (\alpha z)}{1+z^{2}} d z=\pi \cosh (\alpha) \quad(\alpha>0)$

The contour integral could either evaluated directly with $f_{0}(z)=\frac{\cos (\alpha z)}{1+z^{2}}$ 
Clear $[\mathbf{z}, f, \alpha]$;

$f_{0}:=\frac{\operatorname{Cos}[\alpha z]}{\left(z^{2}+1\right)} ;$

$\mathcal{J}_{0}=$ ContourIntegral $\left[f_{0}, \mathbf{z},\{2\},\{\}, "\right.$ Off" $]$

(* pole $\mathbf{z}_{2}=$ i $*$ )

$f(z)=\frac{\cos (\alpha z)}{z^{2}+1}$ has poles with multiplicity

$\left\{\left\{z_{i}, \mu_{i}\right\}, \ldots\right\}=\{\{-\dot{i}, 1\},\{\dot{i}, 1\}\} \Leftrightarrow$ residues $=\left\{\frac{1}{2} \dot{i} \operatorname{Cosh}[\alpha],-\frac{1}{2} \dot{i} \operatorname{Cosh}[\alpha]\right\}$

Residues $\left.\sum_{i} \operatorname{Res}(f(z))\right|_{z=z_{i}}=-\frac{1}{2} \dot{i} \cosh (\alpha)$ for $i=\{2\}$ poles

Contour-integral $\mathcal{J}=\oint \frac{\operatorname{Cos}[z \alpha]}{1+z^{2}} d z=\pi \operatorname{Cosh}[\alpha]$

$\pi \operatorname{Cosh}[\alpha]$

or by decomposition of $f_{0}(z)$ into two parts $f_{1}(z), f_{2}(x)$ with exponential functions $e^{ \pm i \alpha z}$. Adding the two parts $\mathcal{J}_{1,2}$ gives the some result as $\mathcal{J}_{\mathbf{0}}$.

$f_{1}:=\frac{e^{\dot{i} \alpha z}}{2\left(z^{2}+1\right)} ; \quad f_{2}:=\frac{e^{-i \dot{i} \alpha z}}{2\left(z^{2}+1\right)}$

$\mathcal{J}_{1}=$ ContourIntegral $\left[f_{1}, \mathbf{z},\{2\},\{\}\right.$, "No"]

$\mathcal{J}_{2}=$ ContourIntegral $\left[f_{2}, \mathbf{z},\{2\},\{\}\right.$, No"]

fs @ $\left(\mathcal{J}_{1}+\mathcal{J}_{2}\right)===\mathcal{J}_{0}$ 
Contour-integral $\mathcal{J}=\oint \frac{e^{\dot{1} z \alpha}}{2\left(1+z^{2}\right)} d z=\frac{e^{-\alpha} \pi}{2}$

$\frac{e^{-\alpha} \pi}{2}$

Contour-integral $\mathcal{J}=\oint \frac{e^{-i \mathrm{z} \alpha}}{2\left(1+\mathrm{z}^{2}\right)} \mathrm{dl} z=\frac{\mathrm{e}^{\alpha} \pi}{2}$

$\frac{e^{\alpha} \pi}{2}$

True

Example 24 : $\int_{-\infty}^{+\infty} \frac{\sin (z)}{z} d z=\pi$

Example 25: $\oint_{\gamma} \frac{\sin (z)}{\left(z^{2}+1\right)^{4}(z+3 i)^{3}} d z=\frac{3 \pi\left(23+283 e^{2}+13 e^{4}-7 e^{6}\right)}{32768 e^{3}}$

$f(z)=\frac{\sin (z)}{\left(z^{2}+1\right)^{4}(z+3 i)^{3}}$ is integrated with contour $\gamma$ (oriented counter-clockwise) which encircles the triple pole $z_{2}=-\mathbf{3} i$ and fourth order poles $z_{1,3}=\mp i$. Because $\sin (\mathbf{z})$ is holomorphic in $\mathbb{C}$ then $f(z)$ is holomorphic apart from the zeros of the denominator polynomial.

$\mathcal{J}=$ ContourIntegral $\left[\frac{\operatorname{Sin}[z]}{\left(z^{2}+1\right)^{4}(z+3 i)^{3}}, z, A l l,\{\}, " O f f "\right] / / \operatorname{TrigToExp} / / \operatorname{sf}$ 


$f(z)=\frac{\sin (z)}{(z+3 \dot{i})^{3}\left(z^{2}+1\right)^{4}}$ has poles with multiplicity
$\left\{\left\{z_{i}, \mu_{i}\right\}, \ldots\right\}=\{\{-\dot{i}, 4\},\{-3 \dot{i}, 3\},\{\dot{i}, 4\}\} \Leftrightarrow \operatorname{residues}=$
$\left\{-\frac{\dot{\mathbb{i}}(14 \operatorname{Cosh}[1]-9 \sinh [1])}{1536},-\frac{3 \dot{i}(8 \operatorname{Cosh}[3]-15 \operatorname{Sinh}[3])}{32768},-\frac{\dot{\mathbb{i}}(436 \operatorname{Cosh}[1]-639 \operatorname{Sinh}[1])}{98304}\right\}$

$\left.\operatorname{Residues} \sum_{i} \operatorname{Res}(f(z))\right|_{z=z_{i}}=-\frac{3 \dot{i}(-15(9 \sinh (1)+\sinh (3))+148 \cosh (1)+8 \cosh (3))}{32768}$ for $i=$ All poles

Contour-integral $\mathcal{J}=\oint \frac{\operatorname{Sin}[z]}{(3 \dot{i}+z)^{3}\left(1+z^{2}\right)^{4}} d z=\frac{3 \pi(148 \operatorname{Cosh}[1]+8 \operatorname{Cosh}[3]-15(9 \operatorname{Sinh}[1]+\operatorname{Sinh}[3]))}{16384}$

$\frac{3\left(23+283 e^{2}+13 e^{4}-7 e^{6}\right) \pi}{32768 e^{3}}$

Example 26: $\oint_{\gamma} \frac{\sin (z)^{4}}{\left(z-\frac{\pi}{2}\right)^{3}} d z=-4 \pi \overline{0}$

Example 27 : $\oint_{\gamma} \frac{e^{i z}}{1+z^{2}+z^{4}} d z=\frac{\pi}{3} e^{-\frac{\sqrt{3}}{2}}\left(\sqrt{3} \cos \left(\frac{1}{2}\right)+3 \sin \left(\frac{1}{2}\right)\right)$ 
Example 28: $\oint_{\gamma} \frac{e^{i z}}{e^{z}-1} d z=i \frac{\sinh (\pi)^{4}}{12 \pi^{3}} \quad(\$ K=-2)$

Note : with the additional global variable $\$ \kappa$ the number of poles can be controlled. Denominators such as $\left(\boldsymbol{e}^{z}-\mathbf{1}\right)=\mathbf{0}$ give rise to a sequence of simple poles of the form $z_{k}=\mathbf{2} \boldsymbol{\pi} \boldsymbol{i} \cdot \boldsymbol{k}$ with additional index $\boldsymbol{k} \in \mathbb{N}$. Thus, e.g. for $\mathbf{\$} \boldsymbol{K}=\mathbf{2}$ the index $\boldsymbol{k}$ give rise to $\{\mathbf{0 , 1 , 2}\}$ whereas for $\mathbf{\$} \kappa=-\mathbf{2}$ the sequence $\boldsymbol{k}=\{\mathbf{- 2}, \mathbf{- 1 , 0 , 1 , 2}\}$ is obtained. Default value is $\$ \boldsymbol{\kappa}=\mathbf{0}$.

$\$ \kappa=0 ;$

ContourIntegral $\left[\frac{e^{\dot{i} z}}{e^{z}-1}, z\right]$;

$(* k=0,1,2 *)$

$f(z)=\frac{\mathbb{e}^{i z}}{\mathbb{e}^{z}-1}$ has poles with multiplicity

$\left\{\left\{z_{i}, \mu_{i}\right\}, \ldots\right\}=\{\{0,1\},\{2 \dot{i} \pi, 1\}\} \Leftrightarrow$ residues $=\left\{\frac{\dot{i}}{2 \pi},-\frac{\dot{i} \mathbb{e}^{-2 \pi}}{2 \pi}\right\}$

Residues $\left.\sum_{i} \operatorname{Res}(f(z))\right|_{z=z_{i}}=\frac{\dot{i}-\dot{i} \mathbb{e}^{-2 \pi}}{2 \pi}$ for $i=$ All poles

Contour-integral $\mathcal{J}=\oint \frac{\mathbb{e}^{\mathbf{i} z}}{-1+\mathbb{e}^{z}} d z=-1+\mathbb{e}^{-2 \pi}$

Table $\left[\right.$ ContourIntegral $\left[\frac{e^{\dot{i} z}}{e^{z}-1}, z, A l 1,\{\}\right.$, No" $\left.],\{\$ \kappa,-1,-5,-1\}\right] / /$ fs

$\$ \kappa=0 ; \quad(*$ reset to default value *)

Contour-integral $\mathcal{J}=\oint \frac{\mathbb{e}^{\dot{i} z}}{-1+\mathbb{e}^{z}} d z=-\frac{\dot{i} \operatorname{Sinh}[\pi]^{2}}{\pi}$ 
Contour-integral $\mathcal{J}=\oint \frac{\mathbb{e}^{i \mathbf{z}}}{-1+\mathbb{e}^{z}} \mathbb{d} z=\frac{i \operatorname{Sinh}[\pi]^{4}}{12 \pi^{3}}$

Contour-integral $J=\oint \frac{\mathbb{e}^{i z}}{-1+\mathbb{e}^{z}} d z=-\frac{i \operatorname{Sinh}[\pi]^{6}}{360 \pi^{5}}$

Contour-integral $\mathcal{J}=\oint \frac{\mathbb{e}^{\dot{i} z}}{-1+\mathbb{e}^{z}} d z=\frac{\dot{i} \operatorname{Sinh}[\pi]^{8}}{20160 \pi^{7}}$

Contour-integral $\mathcal{J}=\oint \frac{\mathbb{e}^{\mathrm{i} z}}{-1+\mathbb{e}^{z}} \mathrm{dl} z=-\frac{\dot{i} \operatorname{Sinh}[\pi]^{10}}{1814400 \pi^{9}}$

$\left\{-\frac{\dot{i} \operatorname{Sinh}[\pi]^{2}}{\pi}, \frac{\dot{i} \operatorname{Sinh}[\pi]^{4}}{12 \pi^{3}},-\frac{\dot{i} \operatorname{Sinh}[\pi]^{6}}{360 \pi^{5}}, \frac{\dot{i} \operatorname{Sinh}[\pi]^{8}}{20160 \pi^{7}},-\frac{\dot{i} \operatorname{Sinh}[\pi]^{10}}{1814400 \pi^{9}}\right\}$

Example 29: $\oint_{\gamma} \frac{e^{i \alpha z}}{\left(z^{2}+1\right)^{2}} d z=\pi(\alpha \cosh (\alpha)-\sinh (\alpha))$

Example 30: $\oint_{\gamma} \frac{z e^{i \alpha z}}{\left(z^{4}+4\right)} d z=-i \pi \sin (\alpha) \sinh (\alpha)$

For $\alpha \neq 0$ the improper integral $\int_{-\infty}^{+\infty} \frac{x \sin (\alpha x)}{\left(x^{4}+4\right)} d x$ is equal to the imaginary part of the contour integral $\operatorname{Im}\left(\oint_{\gamma} \frac{z e^{i \alpha z}}{\left(z^{4}+4\right)} d z\right)$. 
Clear $[z, \alpha] ;$ \$Assumptions $=\alpha \in$ Reals;

$\mathcal{J}_{+}=$ContourIntegral $\left[\frac{z e^{\dot{i} \alpha z}}{\left(z^{4}+4\right)}, z,\{3,4\},\{\}\right.$, No" $]$;

$\mathcal{J}_{-}=$ContourIntegral $\left[\frac{z e^{\dot{i} \alpha z}}{\left(z^{4}+4\right)}, z,\{1,2\},\{\}\right.$, No" $]$;

$\left\{\mathcal{J}_{+}, \mathcal{J}_{-}\right\}$

$\left(\mathcal{T}_{+}+\mathcal{J}_{-}\right) / /$fs

Contour-integral $\mathcal{J}=\oint \frac{\mathbb{e}^{\dot{i} z \alpha} z}{4+z^{4}} d z=\frac{1}{2} \dot{i} e^{-\alpha} \pi \operatorname{Sin}[\alpha]$

Contour-integral $\mathcal{J}=\oint \frac{\mathbb{e}^{\mathrm{i} z \alpha} \mathrm{z}}{4+\mathrm{z}^{4}} \mathrm{dl} z=-\frac{1}{2} \dot{i} \mathbb{e}^{\alpha} \pi \operatorname{Sin}[\alpha]$

$\left\{\frac{1}{2} \dot{\mathbb{1}} \mathbb{e}^{-\alpha} \pi \operatorname{Sin}[\alpha],-\frac{1}{2} \dot{\mathbb{1}} \mathbb{e}^{\alpha} \pi \operatorname{Sin}[\alpha]\right\}$

$-\dot{i} \pi \operatorname{Sin}[\alpha] \operatorname{Sinh}[\alpha]$

ContourIntegral $\left[\frac{z e^{\dot{i} \alpha z}}{\left(z^{4}+4\right)}, z, A l l,\{\}\right.$, "Off" $]$;

$f(z)=\frac{z \mathbb{e}^{i \alpha z}}{z^{4}+4}$ has poles with multiplicity

$\left\{\left\{z_{i}, \mu_{i}\right\}, \ldots\right\}=\left\{\left\{-(-1)^{1 / 4} \sqrt{2}, 1\right\},\left\{-(-1)^{3 / 4} \sqrt{2}, 1\right\},\left\{(-1)^{1 / 4} \sqrt{2}, 1\right\},\left\{(-1)^{3 / 4} \sqrt{2}, 1\right\}\right\} \Leftrightarrow$ residues $=$

$\left\{-\frac{1}{8} \dot{i} \mathbb{e}^{\alpha}(\operatorname{Cos}[\alpha]-\dot{i} \operatorname{Sin}[\alpha]), \frac{1}{8} \dot{i} \mathbb{e}^{\alpha}(\operatorname{Cos}[\alpha]+i \sin [\alpha]), \frac{1}{8} \mathbb{e}^{-\alpha}(-\dot{i} \operatorname{Cos}[\alpha]+\operatorname{Sin}[\alpha]), \frac{1}{8} \mathbb{e}^{-\alpha}(i \cos [\alpha]+\operatorname{Sin}[\alpha])\right\}$ 
Residues $\left.\sum_{i} \operatorname{Res}(f(z))\right|_{z=z_{i}}=-\frac{1}{4} \mathbb{e}^{-\alpha}\left(\mathbb{e}^{2 \alpha}-1\right) \sin (\alpha)$ for $i=$ All poles

Contour-integral $\mathcal{J}=\oint \frac{\mathbb{e}^{i \mathrm{z} \alpha} z}{4+\mathrm{z}^{4}} d z=-\dot{i} \pi \operatorname{Sin}[\alpha] \operatorname{Sinh}[\alpha]$

Example 31: $\oint_{V} \frac{\cos (\mathrm{z})}{\mathrm{z}(\mathrm{z}-\pi)} d \mathrm{z}=-2 i \quad(|\mathrm{z}| \leq 3)$

Example 32 : $\oint_{\gamma} \frac{e^{\mathrm{z}}}{\mathrm{z}(\mathrm{z}-i \pi)} d \mathrm{z}=-2 \quad(|\mathrm{z}| \leq 3)$

Example 33 : $\oint_{V} \frac{\mathbb{e}^{z}}{z^{2}} d z=2 \pi \pi \quad(|z| \leq 3)$

Example 34: $\oint_{V} \frac{e^{\mathrm{z}}}{(\mathrm{z}-1)(\mathrm{z}-2)} d \mathrm{z}=2 \pi \bar{j}\left(e^{2}-e\right) \quad(|\mathrm{z}| \leq 3)$

Example 35: $\oint_{V} \frac{1-\cos (\mathrm{z})}{\mathrm{z}^{2}} d \mathrm{z}=0 \quad(|\mathrm{z}| \leq 3)$

Example 36: $\oint_{Y} \frac{1}{\mathrm{z}^{2} \sin (\mathrm{z})} d \mathrm{z}=\frac{\pi}{3} i \quad(|\mathrm{z}| \leq 3)$

Example 37: $\oint_{V} \mathrm{z} \cos \left(\frac{1}{\mathrm{z}}\right) d \mathrm{z}=i \pi \quad(|\mathrm{z}| \leq 3)$

For the integrand $f(z)=z \cos \left(\frac{1}{z}\right)$ a change of variable is made by the substitution $z \rightarrow \frac{1}{\zeta}$ together with the differential $d z \rightarrow D t\left(\frac{1}{\zeta}, \zeta\right) d \zeta$. 
Clear $[f, \zeta]$;

$f\left[z_{-}\right]:=z \operatorname{Cos}\left[\frac{1}{z}\right] ;$ changeVar $\left[z_{-}, \zeta_{-}\right]:=\left\{z \rightarrow \frac{1}{\zeta}, \mathbb{d} z \rightarrow \operatorname{Dt}\left[\frac{1}{\zeta}, \zeta\right] d \zeta\right\} ;$

$f[z] d z / /$. changeVar $[z, \zeta]$

$-\frac{\cos [\zeta] d \zeta}{\zeta^{3}}$

The result of this substitution is a pole of third order with respect to $\zeta$.

ContourIntegral $\left[-\frac{\operatorname{Cos}[\zeta]}{\zeta^{3}}, \zeta, A l l,\{\}, "\right.$ Off" $] ; \quad(* z \rightarrow 1 / \zeta *)$

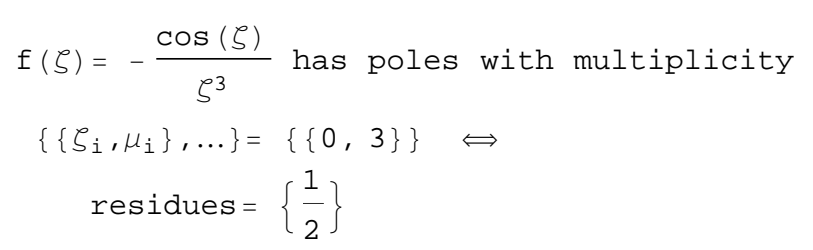

- Product of rational and hyperbolic functions $f(z)=\frac{p(z)}{q(z)} \cdot\left\{\begin{array}{l}\cosh (\alpha z) \\ \sinh (\alpha z)\end{array}\right.$

Furthermore, for products of rational and hyperbolic functions the residue theorem and contour integration can be applied too. 


\section{$\mathcal{J}=\int_{-\infty}^{+\infty} \frac{p(x)}{q(x)} \cdot\left\{\begin{array}{c}\cosh (\alpha x) \\ \sinh (\alpha x)\end{array} d x \quad\right.$ or $\quad \mathcal{J}=\int_{-\infty}^{+\infty} \frac{p(x)}{q(x)} e^{\alpha x} d x$}

Example 38 : $\oint_{V} \frac{\cosh (z)}{z^{2}+\pi^{2}} d z=-1$

Example 39 : $\oint_{V} \frac{\sinh (z)}{z^{4}} d z=\frac{\pi}{3} \bar{\sigma}$

There exists a fourth order pole $\boldsymbol{z}_{\mathbf{1}}=\mathbf{0}$.

ContourIntegral $\left[\frac{\operatorname{Sinh}[z]}{z^{4}}, z, A l 1,\{\}, "\right.$ Off" $]$;

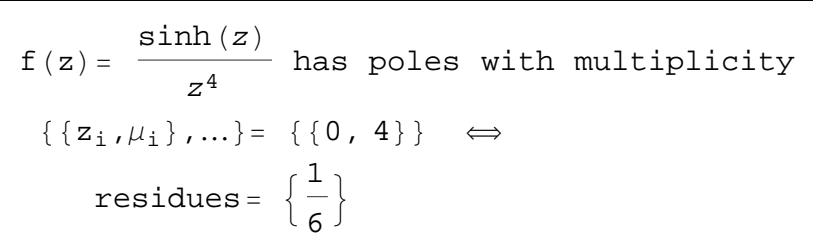

Example 40: $\oint_{V} \frac{\sinh (z)^{\mathrm{n}}}{\mathrm{z}^{\mathrm{n}+1}} d \mathrm{z}=2 \pi i \quad(n \in \mathbb{N})$,

Example 41: $\oint_{V} \frac{\cosh \left(z^{2 n-1}\right.}{z^{2 n-1}} d z=2 \pi i \cdot\left\{1, \frac{3}{2}, \frac{65}{24}, \frac{3787}{720}, \ldots\right\} \quad(n \in \mathbb{N})$ 
Similarly, for $\frac{\cosh (\mathbf{z})^{2 n-1}}{\mathbf{z}^{\mathrm{n}-1}}$ one obtains $2 \pi i \times \mathbf{p}$ with rational numbers $\mathbf{p} \in \mathbb{Q}$

Table $\left[\right.$ ContourIntegral $\left.\left[\frac{\operatorname{Cosh}[z]^{2 n-1}}{z^{2 n-1}}, z, A l l,\{\}, " N o "\right],\{n, 1,9\}\right]$

Contour integral $\mathcal{J}=\oint \frac{\operatorname{Cosh}[z]}{z} d z=2$ i $\pi$

Contour integral $\mathcal{J}=\oint \frac{\operatorname{Cosh}[z]^{3}}{z^{3}} d z=3 \dot{i} \pi$

Contour integral $\mathcal{J}=\oint \frac{\operatorname{Cosh}[z]^{5}}{z^{5}} d z=\frac{65 \text { i } \pi}{12}$

Contour integral $\mathcal{J}=\oint \frac{\operatorname{Cosh}[z]^{7}}{z^{7}} d z=\frac{3787 \dot{i} \pi}{360}$

Contour integral $\mathcal{J}=\oint \frac{\operatorname{Cosh}[z]^{9}}{z^{9}} d z=\frac{9509 \dot{i} \pi}{448}$

Contour integral $\mathcal{J}=\oint \frac{\operatorname{Cosh}[z]^{11}}{z^{11}} d z=\frac{79549811 \dot{i} \pi}{1814400}$

Contour integral $\mathcal{J}=\oint \frac{\operatorname{Cosh}[z]^{13}}{z^{13}} d z=\frac{22036379521 \text { i } \pi}{239500800}$ 
Contour integral $\mathcal{J}=\oint \frac{\operatorname{Cosh}[z]^{15}}{z^{15}} d z=\frac{567547087381 \text { i } \pi}{2905943040}$

Contour integral $\mathcal{J}=\oint \frac{\operatorname{Cosh}[z]^{17}}{z^{17}} d z=\frac{624922249423799 \text { i } \pi}{1494484992000}$

$\left\{2 \dot{i} \pi, 3 \dot{i} \pi, \frac{65 \dot{i} \pi}{12}, \frac{3787 \dot{i} \pi}{360}, \frac{9509 \dot{i} \pi}{448}, \frac{79549811 \dot{\mathbf{i}} \pi}{1814400}, \frac{22036379521 \dot{\mathbf{i}} \pi}{239500800}, \frac{567547087381 \dot{i} \pi}{2905943040}, \frac{624922249423799 \dot{\mathbf{1}} \pi}{1494484992000}\right\}$

- Rational functions with trigonometric and hyperbolic functions $F(\sin (\theta), \cos (\theta))$ or $F(\sinh (\theta), \cosh (\theta))(E x a m p l e s 42-57)$

Another type of integrand consists of rational functions which are combinations of trigonometric or hyperbolic functions.

$$
\begin{array}{ll}
\int_{0}^{2 \pi} F(\sin (\theta), \cos (\theta)) d \theta=\oint_{\gamma} \frac{1}{i z} F\left(\frac{(z-1 / z)}{2 i}, \frac{(z+1 / z)}{2}\right) d z & \left(z=e^{i \theta}\right) \\
\int_{0}^{2 \pi} F(\sinh (\theta), \cosh (\theta)) d \theta=\oint_{\gamma} \frac{1}{z} F\left(\frac{(z-1 / z)}{2}, \frac{(z+1 / z)}{2}\right) d z \quad\left(z=e^{\theta}\right)
\end{array}
$$

Typically, $\boldsymbol{F}$ is a rational function the arguments of which are trigonometric or hyperbolic functions. Here, $\boldsymbol{\theta} \in\{\mathbf{0}, \mathbf{2} \pi\}$.

For trigonometric functions with $z=e^{i \theta}$ and $\frac{1}{z}=e^{-i \theta} \quad$ substitution yields $\left\{\cos (\theta)=\frac{(z+1 / z)}{2}, \sin (\theta)=\frac{(z-1 / z)}{2 i}, d \theta=\frac{d z}{i z}\right\}$; $\operatorname{similarly}$

for hyperbolic functions with $z=e^{\theta}$ and $\frac{1}{z}=e^{-\theta}$ corresponding substitution is $\left\{\cosh (\theta)=\frac{(z+1 / z)}{2}, \sinh (\theta)=\frac{(z-1 / z)}{2}, d \theta=\frac{d z}{z}\right\}$.

The following substitution rules will be used for all integrands of type $\mathbf{F}(\sin (\theta), \cos (\theta))$ respectively $\mathbf{F}(\sinh (\theta), \cosh (\theta))$; they $\operatorname{are}$ not included in the package ContourIntegration 


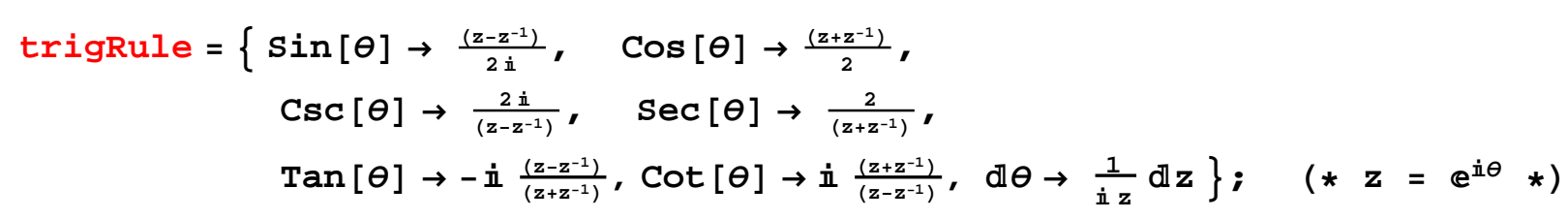

hypRule $=\left\{\sinh [\theta] \rightarrow \frac{\left(z-z^{-1}\right)}{2}, \operatorname{Cosh}[\theta] \rightarrow \frac{\left(z+z^{-1}\right)}{2}\right.$,

$\operatorname{Csch}[\theta] \rightarrow \frac{2}{\left(z-z^{-1}\right)}, \operatorname{Sech}[\theta] \rightarrow \frac{2}{\left(z+z^{-1}\right)}$,

$\left.\operatorname{Tanh}[\theta] \rightarrow \frac{\left(z-z^{-1}\right)}{\left(z+z^{-1}\right)}, \operatorname{Coth}[\theta] \rightarrow \frac{\left(z+z^{-1}\right)}{\left(z-z^{-1}\right)}, d \theta \rightarrow \frac{1}{z} d z \quad\right\} ; \quad\left(* z=e^{\theta} *\right)$

Substitutions applied to the original integrand (with trigonometric or hyperbolic functions) will result in a rational polynomial in $z$ for which residues can be calculated.

Example 42: $\int_{0}^{2 \pi} \frac{1}{5+\sin (\theta)} d \theta=\oint_{\gamma} \frac{1}{2 z^{2}+5 i z-2} d z=\frac{2 \pi}{3}$

Example 43: $\oint_{\gamma} \frac{1}{\cos (\theta)} d \theta=\frac{1}{i} \oint_{\gamma} \frac{2}{\mathrm{z}^{2}+1} d \mathrm{z}=0$

Example 44: $\int_{0}^{2 \pi} \frac{1}{\mathrm{a}+\cos (\theta)} d \theta=\frac{2}{i} \oint_{\gamma} \frac{1}{z^{2}+2 a z+1} d z=\frac{2 \pi}{\sqrt{\mathrm{a}^{2}-1}}$

Example 45: $\int_{0}^{\pi} \frac{1}{(a+\cos (\theta))^{2}} d \theta=\frac{2}{\tilde{b}} \oint_{Y} \frac{1}{\left(z^{2}+2 a z+1\right)^{2}} d z=\frac{a \pi}{\left(a^{2}-1\right)^{3 / 2}}$

Consider $\int_{0}^{\pi} \frac{1}{(a+\cos (\theta))^{2}} d \theta$ for $\mathbf{a}>1$. Since $\cos (\theta)$ is an even function thus $\frac{1}{2} \int_{0}^{2 \pi} \frac{1}{(a+\cos (\theta))^{2}} d \theta$ so that the contour integral is $\frac{2}{i} \oint_{\gamma} \frac{z}{\left(z^{2}+2 a z+1\right)^{2}} d z$. 
Clear[F4, f4, a] ;

\$Assumptions $=a>1$;

$\mathbf{F} 4\left[\theta_{-}\right]:=\frac{1}{(a+\operatorname{Cos}[\theta])^{2}} ; \quad f 4\left[z_{-}\right]:=\left(\frac{F 4[\theta]}{\text { in } z} /\right.$. trigRule $) / /$ sf

$\mathbf{f}[\mathbf{z}] / / \mathrm{tF} / / \mathbf{s F}$

$-\frac{4 \dot{i} z}{\left(2 a z+z^{2}+1\right)^{2}}$

The denominator has two double roots :

$\zeta=$ Solve $[$ Denominator $[f 4[z]]=0, z] / /$. $\left\{\operatorname{Rule}\left[a_{-}, b_{-}\right] \rightarrow\right.$ List $\left.[b]\right\} / /$ Union //Flatten

$\left\{-a-\sqrt{-1+a^{2}},-a+\sqrt{-1+a^{2}}\right\}$

The root $z_{1}=-a-\sqrt{a^{2}-1}$ is located outside the unit circle because $\mathbf{a}>\mathbf{1}$. Thus, only the double pole $z_{2}=a-\sqrt{a^{2}-1}$ will contribute to the contour integral.

ContourIntegral $[f 4[z], z,\{2\},\{\}, " N o "]$

Contour integral $\mathcal{J}=\oint-\frac{4 \dot{i} z}{\left(1+2 a z+z^{2}\right)^{2}} d z=\frac{2 \pi a}{\left(a^{2}-1\right)^{3 / 2}}$

$\frac{2 a \pi}{\left(-1+a^{2}\right)^{3 / 2}}$

Direct evaluation of the integral $\int_{0}^{\pi} \frac{1}{(a+\cos (\theta))^{2}} d \theta$ confirms the value found by contour integration.

$\int_{0}^{\pi} F 4[\theta] d \theta / / t F$ 
$\frac{\pi a}{\left(a^{2}-1\right)^{3 / 2}}$

Example 46: $\int_{0}^{2 \pi} \frac{1}{1+b \cos (\theta)} d \theta=\frac{2}{i} \oint_{\gamma} \frac{1}{\left(b z^{2}+2 z+b\right)} d z=\frac{2 \pi}{\sqrt{1-b^{2}}}$

$(|\mathbf{b}|<\mathbf{1})$

Example $47: \int_{0}^{2 \pi} \frac{1}{1+\sin (\theta)^{2}} d \theta=\oint_{\gamma} \frac{4 i z}{z^{4}-6 z^{2}+1} d z=\sqrt{2} \pi$

Clear $[F 6, f 6]$

$\mathrm{F} 6\left[\theta_{-}\right]:=\frac{1}{1+\operatorname{Sin}[\theta]^{2}} ; \quad f 6\left[z_{-}\right]:=\left(\frac{F 6[\theta]}{\text { in }} /\right.$. trigRule $) / / \mathbf{s f}$

$\mathrm{f} 6[\mathrm{z}] / / \mathrm{tF} / / \mathrm{sF}$

$\frac{4 \dot{i} z}{z^{4}-6 z^{2}+1}$

ContourIntegral $[f 6[z], z,\{2,3\},\{\}, "$ Off" $] / / f s$

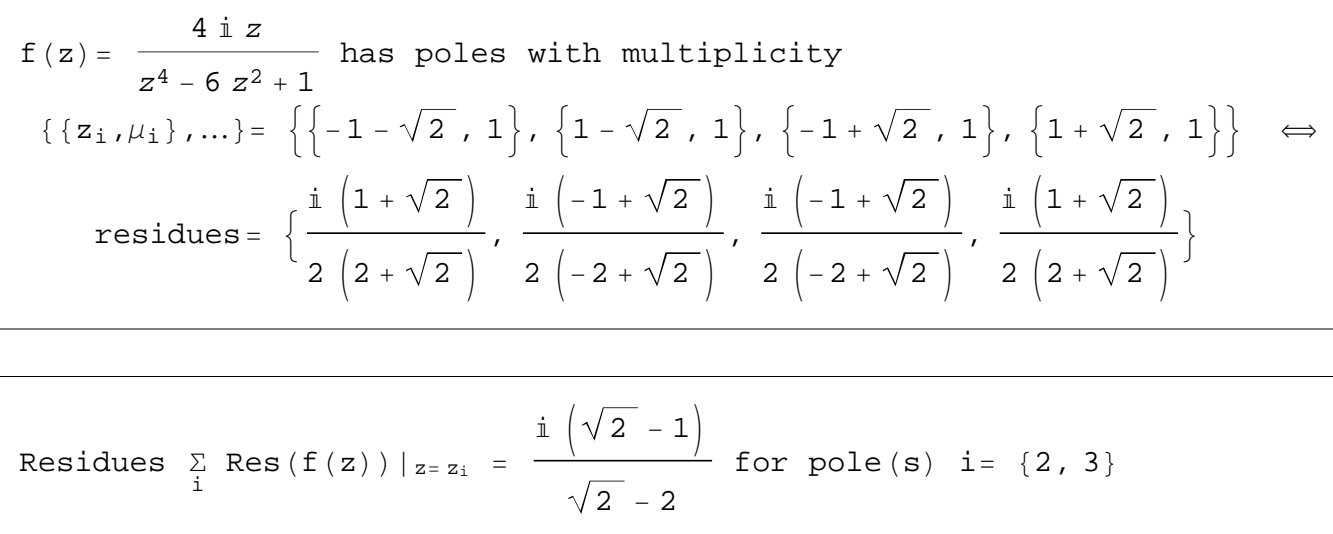


Contour integral $\mathcal{J}=\oint \frac{4 \dot{i} z}{1-6 z^{2}+z^{4}} d z=-\frac{2(\sqrt{2}-1) \pi}{\sqrt{2}-2}$

$\sqrt{2} \pi$

Direct evaluation of the integral $\int_{0}^{2 \pi} \frac{1}{1+\sin (\theta)^{2}} d \theta$ confirms the value found by contour integration.

$\int_{0}^{2 \pi} F 6[\theta] d \theta$

$\sqrt{2} \pi$

Example 48 : $\int_{0}^{2 \pi} \frac{1}{\left(\mathrm{a}+\mathrm{b} \cos (\theta)^{2}\right)^{2}} d \theta=\oint_{C} \frac{-16 i z^{3}}{\left(4 a z^{2}+b\left(z^{2}+1\right)^{2}\right)^{2}} d z=\frac{(2 \mathrm{a}+\mathrm{b}) \pi}{(\mathrm{a}(\mathrm{a}+\mathrm{b}))^{3 / 2}}$

Clear[F7, f7, a, b] ;

\$Assumptions $=\{a>0, b>0\} ;$

F7 $\left[\theta_{-}\right]:=\frac{1}{\left(a+b \operatorname{Cos}[\theta]^{2}\right)^{2}} ; \quad f 7\left[z_{-}\right]:=\left(\frac{F 7[\theta]}{\text { ir }} /\right.$. trigRule $) / /$ sf

f7[z] // ExpandAll // fs // tF // sF

$-\frac{16 \dot{1} z^{3}}{\left(4 a z^{2}+b\left(z^{2}+1\right)^{2}\right)^{2}}$

ContourIntegral[f7[z], z, \{3, 4\}, \{\}, "No"] ;

Contour integral $\mathcal{J}=\oint-\frac{\dot{i}}{z\left(a+\frac{1}{4} b\left(\frac{1}{z}+z\right)^{2}\right)^{2}} d z=\frac{\pi(2 a+b)}{(a(a+b))^{3 / 2}}$ 
Direct evaluation of the integral $\int_{0}^{2 \pi} \frac{1}{\left(a+b \cos (\theta)^{2}\right)^{2}} d \theta$ confirms the value found by contour integration.

$\int_{0}^{2 \pi} F 7[\theta] d \theta$

$\frac{(2 a+b) \pi}{(a(a+b))^{3 / 2}}$

Example 49: $\int_{0}^{2 \pi} \frac{\cos (3 \theta)^{2}}{5-4 \cos (2 \theta)} d \theta=\oint_{\gamma} \frac{i\left(z^{6}+1\right)^{2}}{4 z^{5}\left(2 z^{4}-5 z^{2}+2\right)} d z=\frac{3 \pi}{8}$

Example 50 : $\int_{0}^{2 \pi} \frac{1}{1-2 \mathrm{a} \cos (\theta)+\mathrm{a}^{2}} d \theta=\oint_{\gamma} \frac{-i}{-a z^{2}+\left(a^{2}+1\right) z-a} d z=\frac{\pi}{\mathrm{a}^{2}-1} \quad(\mathrm{a} \neq \pm \mathbf{1})$

Example $51: \int_{0}^{2 \pi} \frac{\cos (3 \theta)^{2}}{1-2 \mathrm{a} \cos (\theta)+\mathrm{a}^{2}} d \theta=\oint_{\gamma} \frac{-i\left(z^{6}+1\right)^{2}}{4 z^{6}(a-z)(a z-1)} d z=\frac{\pi\left(\mathrm{a}^{6}+1\right)}{\mathrm{a}^{6}\left(\mathrm{a}^{2}-1\right)} \quad(\mathrm{a} \neq \pm 1)$

Clear [F10, f10, a];

\$Assumptions $=((-1>a|| a \geq 1) \& \& a \in$ Reals $)$;

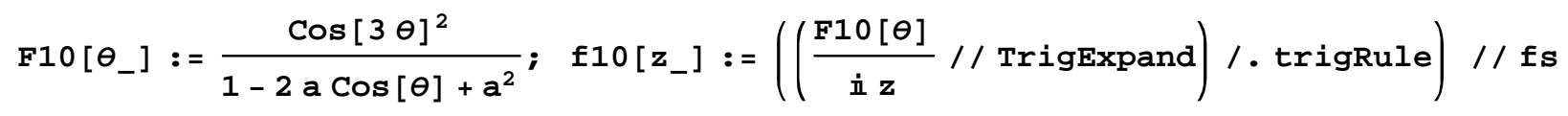

f10[z] // ExpandAll // fs // tF // SF

$-\frac{\dot{\mathbb{1}}\left(z^{6}+1\right)^{2}}{4 z^{6}(a-z)(a z-1)}$

ContourIntegral[f10[z], z, \{1, 2\}, \{\}, "No"] ;

Contour integral $\mathcal{J}=\oint-\frac{\dot{1}\left(1+z^{6}\right)^{2}}{4(a-z) z^{6}(-1+a z)} d z=\frac{\pi\left(a^{6}+1\right)}{a^{6}\left(a^{2}-1\right)}$ 
Direct evaluation of the integral $\int_{0}^{2 \pi} \frac{\cos (3 \theta)^{2}}{1-2 a \cos (\theta)+a^{2}} d \theta$ confirms the value found by contour integration.

$\left(\int_{0}^{2 \pi} \mathrm{F} 10[\theta] d \theta / /\right.$. rule 1$) / /$ rule2 $/ / \mathrm{sf} / / \mathrm{tF}$

$\frac{\pi\left(a^{6}+1\right)}{a^{6}\left(a^{2}-1\right)}$

Example $52: \int_{0}^{2 \pi} \frac{\cos (2 \theta)}{1-2 \mathrm{a} \cos (\theta)+\mathrm{a}^{2}} d \theta=\oint_{C} \frac{-i\left(z^{4}+1\right)}{2 z^{2}(a-z)(a z-1)} d z=\frac{2 \pi}{\mathrm{a}^{2}\left(\mathrm{a}^{2}-1\right)} \quad(\mathrm{a} \neq \pm 1)$

Example $53: \oint_{\gamma} \tan (\zeta) d \zeta=\frac{1}{i} \oint_{\gamma} \frac{z^{2}-1}{z^{3}+z} d z=2 \pi$

Example 54: $\int_{0}^{2 \pi} \tan (\theta+i \mathrm{a}) d \theta=\oint_{\gamma} \frac{e^{2 a}-z^{2}}{e^{2 a} z+z^{3}} d z=-2 \pi \bar{t} \quad(\mathbf{a} \in \mathbb{R})$

Clear[F13, f13, a];

\$Assumptions $=(a \in$ Reals $)$;

F13 $\left[\theta_{-}\right]:=\operatorname{Tan}\left[\theta+\dot{i} a_{0}\right] \quad f 13\left[z_{-}\right]:=\left(\left(\frac{F 13[\theta]}{i \mathbf{z}} / /\right.\right.$ TrigExpand $) /$ trigRule $) / /$ fs $/ /$ ExpandAll // Together // sf f13 [z]

$\frac{e^{2 a}-z^{2}}{e^{2 a} z+z^{3}}$

ContourIntegral[f13[z], z, All, \{\}, "Off"] ; 
$f(z)=\frac{e^{2 a}-z^{2}}{\mathbb{e}^{2 a} z+z^{3}}$ has poles with multiplicity

$\left\{\left\{z_{i}, \mu_{i}\right\}, \ldots\right\}=\left\{\{0,1\},\left\{-\dot{i} \mathbb{e}^{a}, 1\right\},\left\{\dot{i} \mathbb{e}^{a}, 1\right\}\right\} \Leftrightarrow$

residues $=\{1,-1,-1\}$

Residues $\left.\sum_{i} \operatorname{Res}(f(z))\right|_{z=z_{i}}=-1$ for pole(s) $i=$ All

Contour integral $\mathcal{J}=\oint \frac{e^{2 a}-z^{2}}{e^{2 a} z+z^{3}} d z=-2 \dot{i} \pi$

Direct evaluation of the integral $\int_{0}^{2 \pi} \tan (\theta+i \boldsymbol{a}) d \theta$ confirms the value found by contour integration.

$\int_{0}^{2 \pi} F 13[\theta] d \theta$

$-2 \dot{1} \pi$

Example 55: $\int_{0}^{\pi} e^{i n \theta}(\cos (n \theta)-\sin (n \theta))^{3} d \theta \stackrel{n \rightarrow-2}{=} \oint_{\gamma} \frac{-(1-\dot{i})\left(\dot{i}+z^{4}\right)^{3}}{4 z^{9}} d z=\frac{3}{2}(1-\tilde{i}) \pi \quad(n \in \mathbb{Z})$

Example 56: $\oint_{V} \frac{1}{\sinh (2 \theta)} d \theta=\oint_{V} \frac{2 \mathrm{z}}{\mathrm{z}^{4}-1} d \mathrm{z}=i \pi$

$(|\mathbf{z}|>1)$

Note : TrigExpand is applicable with respect to trigonometric and hyperbolic functions as well. In addition the substitution rule hypRule is used to convert hyperbolic functions into $f(z)$ using $z=\boldsymbol{e}^{\theta}$. 
ContourIntegration_Pl.nb

$$
\begin{aligned}
& \text { Clear[F15, f15] ; } \\
& \text { F15 }\left[\theta_{-}\right]:=\frac{1}{\operatorname{Sinh}[2 \theta]} ; \quad f 15\left[z_{-}\right]:=\left(\left(\frac{F 15[\theta]}{z} / / \text { TrigExpand }\right) / . \text { hypRule }\right) \\
& f 15[z] / / s f / / t F / / s F \\
& \frac{2 z}{z^{4}-1}
\end{aligned}
$$

All four poles are comprised by the contour $\gamma$ with radius $\boldsymbol{R}>\mathbf{1}$. If the contour $\boldsymbol{\gamma}$ is restricted to $\mathbf{H}_{+}$then a non-zero value results for the contour antegal.

ContourIntegral $[f 15[z], z,\{1,3,4\},\{\}, " O f f "]$

$$
\begin{aligned}
& f(z)=\frac{2}{z\left(z-\frac{1}{z}\right)\left(z+\frac{1}{z}\right)} \text { has poles with multiplicity } \\
& \left\{\left\{z_{i}, \mu_{i}\right\}, \ldots\right\}=\{\{-1,1\},\{-\dot{1}, 1\},\{1,1\},\{\dot{i}, 1\}\} \Leftrightarrow \\
& \quad \text { residues }=\left\{\frac{1}{2},-\frac{1}{2}, \frac{1}{2},-\frac{1}{2}\right\}
\end{aligned}
$$

Residues $\left.\sum_{i} \operatorname{Res}(f(z))\right|_{z=z_{i}}=\frac{1}{2}$ for $\operatorname{pole}(s) i=\{1,3,4\}$

Contour integral $\mathcal{J}=\oint \frac{2}{\mathrm{z}\left(-\frac{1}{z}+\mathrm{z}\right)\left(\frac{1}{z}+\mathrm{z}\right)} \mathrm{d} \mathrm{z}=\dot{\mathrm{i}} \pi$

i $\pi$

For the indefinite integral using Integrate together with rule 3 there is

171 
$\operatorname{rule} 3=\left\{\log \left[\mathbf{A}_{-}\right]-\log \left[\mathbf{B}_{-}\right] \rightarrow \log \left[\mathbf{A}_{/} \mathbf{B}\right], \mathbf{A}_{-} \log \left[\mathbf{B}_{-}\right] \rightarrow \log \left[\mathbf{B}^{\mathbf{A}}\right]\right\} ;$

F15a $[\theta]=F 15[\theta] / /$ TrigExpand;

$\left(\int \mathrm{F} 15 \mathrm{a}[\theta] \mathrm{d} \theta / / \mathrm{fs}\right) / /$. rule3

$\log [\sqrt{\operatorname{Tanh}[\theta]}]$

Example 57: $\oint \tanh (3 \theta) d \theta=\oint_{\gamma} \frac{z^{6}-1}{z^{7}+z} d z=2 \pi i$

Clear $[F 16, f 16]$;

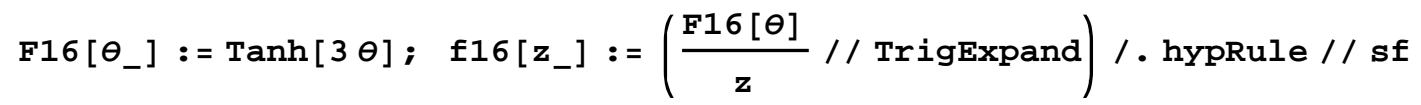

$f 16[z] / / t F$

$\frac{z^{6}-1}{z^{7}+z}$

The denominator gives rise to a single pole $z_{1}=\mathbf{0}$ in addition to three complex-conjugated poles $z_{2,3}=\mp i, \quad z_{4,5}=\mp \frac{\sqrt{3}}{2} \mp \frac{i}{2}$ and $z_{6,7}= \pm \frac{\sqrt{3}}{2} \mp \frac{i}{2}$. All poles are located within a circular contour $\gamma$ of radius $\boldsymbol{R}>\mathbf{3}$.

ContourIntegral[f16[z], z, All, \{\}, "Off"];

$f(z)=\frac{z^{6}-1}{z^{7}+z}$ has poles with multiplicity

$\left\{\left\{\mathbf{z}_{i}, \mu_{i}\right\}, \ldots\right\}=\left\{\{0,1\},\{-\dot{\mathbb{1}}, 1\},\left\{-(-1)^{1 / 6}, 1\right\},\left\{-(-1)^{5 / 6}, 1\right\},\{\dot{1}, 1\},\left\{(-1)^{1 / 6}, 1\right\},\left\{(-1)^{5 / 6}, 1\right\}\right\} \Leftrightarrow$ residues $=\left\{-1, \frac{1}{3}, \frac{1}{3}, \frac{1}{3}, \frac{1}{3}, \frac{1}{3}, \frac{1}{3}\right\}$ 
Residues $\left.\sum_{i} \operatorname{Res}(f(z))\right|_{z=z_{i}}=1$ for $\operatorname{pole}(s) \quad i=\operatorname{All}$

Contour integral $\mathcal{J}=\oint \frac{-1+z^{6}}{z+z^{7}} d z=2$ i $\pi$

Using Integrate the value for the indefinite integral is

$\int \mathrm{F} 16[\theta] d \theta$

$\frac{1}{3} \log [\operatorname{Cosh}[3 \theta]]$

\section{- Conclusions}

In conclusion the author is convinced that the package ContourIntegration $\mathbf{m}$ is a useful extension of the built-in procedure Integrate in Mathematica. It is expected that Mathematica users will find the main procedure ContourIntegral and additional routines for determinating poles, and evaluating the corresponding residues helpful.

In a subsequent paper, i.e. the second part, several topics in the context of contour integration will be treated such as :

- Change of integration variables in order to transform improper integrals into contour integrals,

- Creation of all types of contours consisting of polylines and circular arcs with the procedures showPolygonalContour and contourPathGeneration and visualization with contourPathGraphic,

- Determination of those singularities of a given complex function which are located insided a closed contour path $\gamma$,

- Determination of arbitrary branch cuts using showBranchCut and investigation of contour integrals for complex functions with branch cuts,

- Treatment of the integral representation of Meijer G function with an 'exotic' contour path meandering around certain poles of the integrand and excluding others. 


\section{Appendix}

The author is also grateful to V. Gerdt (private communication 4/2016) for suggesting an extension of the algorithm to cases where the exact polynomial roots are not given explicitly but in terms of exact root objects. Consider the modification of Example $16 \oint_{\gamma} \frac{f(z)}{\left(z^{5}-1\right)} d z$ such as $\oint_{\gamma} \frac{f(z)}{\left(z^{5}-5 z-1\right)} d z$.

Instead of the simple poles for the polynomial $z^{5}-1$ in the denominator of the integrand

Solve $\left[z^{5}-1==0, z\right] / .\left\{\right.$ Rule $\left.\left[a_{-}, b_{-}\right] \rightarrow b\right\} / /$ Flatten

$\left\{1,-(-1)^{1 / 5},(-1)^{2 / 5},-(-1)^{3 / 5},(-1)^{4 / 5}\right\}$

one has to deal with the following 5th order polynomial $z^{5}-5 z-1$ whose roots obtained from Solve are given in terms of Root objects.

Solve $\left[z^{5}-5 z-1=0, z\right] / .\left\{\right.$ Rule $\left.\left[a_{-}, b_{-}\right] \rightarrow b\right\} / /$ Flatten

$\left\{\operatorname{Root}\left[-1-5 \# 1+\# 1^{5} \&, 1\right], \operatorname{Root}\left[-1-5 \# 1+\# 1^{5} \&, 2\right]\right.$,

$\left.\operatorname{Root}\left[-1-5 \# 1+\# 1^{5} \&, 3\right], \operatorname{Root}\left[-1-5 \# 1+\# 1^{5} \&, 4\right], \operatorname{Root}\left[-1-5 \# 1+\# 1^{5} \&, 5\right]\right\}$

In order to cope with these situation the routines polesofComplexFunctions and calculateResidues have to be slightly modified. $f[z]$ is an arbitrary complex function which could be

Clear $[\mathbf{f}, \mathbf{z}]$;

polesofComplexFunction $\left[\frac{f[z]}{z^{4}+1}, \mathbf{z}, A l l,\{\}\right.$, No" $]$

$\left\{\left\{-(-1)^{1 / 4},-(-1)^{3 / 4},(-1)^{1 / 4},(-1)^{3 / 4}\right\},\{1,1,1,1\}, F\right\}$

Clear $[\mathrm{f}, \mathbf{z}]$;

polesofComplexFunction $\left[\frac{f[z]}{z^{4}-5 z+1}, z, A l l,\{\}, " N o "\right]$ 
$\{\{-0.918814-1.48481 \dot{i}, 0.200322,1.63731,-0.918814+1.48481 \dot{i}\},\{1,1,1,1\}, F\}$

Clear $[z, f]$

polesofComplexFunction $\left[\frac{f[z]}{z^{5}-1}, \mathbf{z}\right.$, All, \{\}, No" $]$

$\left\{\left\{1,-(-1)^{1 / 5},-(-1)^{3 / 5},(-1)^{2 / 5},(-1)^{4 / 5}\right\},\{1,1,1,1,1\}, F\right\}$

polesofComplexFunction $\left[\frac{f[z]}{z^{5}-5 z-1}, z, A l l,\{\}, " N o "\right]$

$\{\{-1.4405,-0.200064,1.54165,0.0494564-1.49944 \dot{1}, 0.0494564+1.49944 \dot{1}\},\{1,1,1,1,1\}, F\}$

Of course, a numerical representation of the roots of higher order polynomials (given as RootObjects) is obtained in a straightforward way; the calculation of residues or finally the value of the contour integral is given as

Clear $[\mathbf{z}, \mathbf{f}]$

calculateResidues $\left[\frac{f[z]}{z^{4}+1}, z, A l l,\{\}\right.$, No" $] / /$ fs

$\frac{1}{4}(-1)^{1 / 4}\left(\mathrm{f}\left[-(-1)^{1 / 4}\right]-\mathrm{f}\left[(-1)^{1 / 4}\right]+\dot{i}\left(\mathrm{f}\left[-(-1)^{3 / 4}\right]-\mathrm{f}\left[(-1)^{3 / 4}\right]\right)\right)$

Clear $[\mathbf{z}, \mathbf{f}]$;

ContourIntegral $\left[\frac{f[z]}{z^{4}-5 z+1}, z, A l l,\{\}\right.$, "No" ] // Chop

Contour integral $\mathcal{J}=\oint \frac{f[z]}{1-5 z+z^{4}} d z=(0 .+0 . \dot{1})-(0 .+1.26477 \dot{i}) f(0.200322)$

$(0 .-1.26477$ i) $\mathrm{f}[0.200322]$ 


\section{Clear $[\mathbf{z}, \mathbf{f}]$}

ContourIntegral $\left[\frac{f[z]}{z^{5}-1}, z, A l l,\{\}\right.$, No" $] / /$ sf

$$
\begin{aligned}
& \text { Contour integral } \mathcal{J}=\oint \frac{\mathrm{f}[\mathrm{z}]}{-1+\mathrm{z}^{5}} \mathrm{dz}= \\
& \frac{1}{10} \pi\left(4 \dot{\mathrm{i}} f(1)+(\sqrt{10-2 \sqrt{5}}-\dot{\mathrm{i}}(1+\sqrt{5})) f(-\sqrt[5]{-1})-\sqrt{2(5+\sqrt{5})} f\left((-1)^{2 / 5}\right)+\dot{i} \sqrt{5} f\left((-1)^{2 / 5}\right)-\dot{i} f\left((-1)^{2 / 5}\right)+\right. \\
& \left.\quad \sqrt{2(5+\sqrt{5})} f\left(-(-1)^{3 / 5}\right)+\dot{i} \sqrt{5} f\left(-(-1)^{3 / 5}\right)-\dot{i} f\left(-(-1)^{3 / 5}\right)-\sqrt{10-2 \sqrt{5}} f\left((-1)^{4 / 5}\right)-\dot{i} \sqrt{5} f\left((-1)^{4 / 5}\right)-\dot{i} f\left((-1)^{4 / 5}\right)\right)
\end{aligned}
$$

$\frac{1}{10} \pi\left(4 \dot{i} f[1]+(\sqrt{10-2 \sqrt{5}}-\dot{i}(1+\sqrt{5})) \mathbf{f}\left[-(-1)^{1 / 5}\right]-\dot{i} f\left[(-1)^{2 / 5}\right]+\dot{i} \sqrt{5} \mathrm{f}\left[(-1)^{2 / 5}\right]-\sqrt{2(5+\sqrt{5})} \mathrm{f}\left[(-1)^{2 / 5}\right]-\right.$

i $\left.f\left[-(-1)^{3 / 5}\right]+\dot{i} \sqrt{5} \mathrm{f}\left[-(-1)^{3 / 5}\right]+\sqrt{2(5+\sqrt{5})} \mathrm{f}\left[-(-1)^{3 / 5}\right]-\dot{i} f\left[(-1)^{4 / 5}\right]-\dot{i} \sqrt{5} \mathrm{f}\left[(-1)^{4 / 5}\right]-\sqrt{10-2 \sqrt{5}} \mathrm{f}\left[(-1)^{4 / 5}\right]\right)$

\section{Clear $[\mathbf{z}, \mathbf{f}]$;}

ContourIntegral $\left[\frac{f[z]}{z^{5}-5 z-1}, z, A l l,\{\}\right.$, No" $] / /$ Chop // ce

Contour integral $\mathcal{J}=\oint \frac{f[z]}{-1-5 z+z^{5}} d z=(0 .-1.25865 \dot{i}) f(-0.200064)+(0.0503704+0.3041 \dot{i}) f(0.0494564-1.49944 \dot{\mathbb{i}})-$

$(0.0503704-0.3041$ i) $f(0.0494564+1.49944 \dot{i})+(0 .+0.270323 \dot{i}) f(1.54165)+(0 .+0 . \dot{i})$

$0 .+0.0503704 f[0.0494564-1.49944 \dot{i}]-0.0503704 f[0.0494564+1.49944 \dot{i}]+$

$\dot{i}(0 .-1.25865 f[-0.200064]+0.3041 \mathrm{f}[0.0494564-1.49944 \dot{i}]+0.3041 \mathrm{f}[0.0494564+1.49944 \dot{i}]+0.270323 f[1.54165])$ 


\section{- Acknowledgement}

The author wants to thank Paul Abbott for a private communication offering his help for clarification of the notational problem for line integrals [2]. Moreover, the author is particularly grateful to Michael Trott/WRI for his tireless competent support answering - over the period where this project became mature - numerous questions and gave profound advices to subtle technical problems in Mathematica.

\section{- References}

[1] Paul Abbott “Tricks of the Trade; Contour Integration” The Mathematica Journal, Volume 4 Issue 3 (1994) , p. 37-38

[2] Paul Abbott "Tricks of the Trade; Mathematica for Scientists and Engineers" The Mathematica Journal, Volume 6 Issue 3 (1996) , p. 23-24

[3] Thomas Bahder "Mathematica for Scientists and Engineers", Addison-Wesley (1995), Chapt. 6.4.3, p. 318 -323

[4] Michael Trott \& Oleg Marichev from The Wolfram Functions Site : http://functions.wolfram.com/NB/Notations.nb (description of the special contour $\mathcal{L}$ )

[5] Klemens Burg, Herbert Haf \& Friedrich Wille "Funktionentheorie, Höhere Mathematik für Ingenieure, Naturwissenschaftler und Mathematiker", B.G.Teubner Verlag (2004), ISBN 3-519-00480-1, Chapt. 3.2, p. 129

[6] Walter Strampp, Victor Ganzha \& Evgenij Vorozhtsov "Höhere Mathematik mit Mathematica, Bd. 4 : Funktionentheorie, Fourier- und Laplacetransformationen", Vieweg Lehrbuch (1997), ISBN 3-528-06791-8, Chapt. 4.1, p. 100-126

[7] John H. Mathews \& Russell W. Howell “Complex Analysis for Mathematics and Engineering” 6th ed., Jones \& Bartlett Learning (2012), ISBN 978-1-4496-0445-5, Chapt. 8.7, p. 334-343

[8] Alexander O. Gogolin "Lectures on Complex Integration", Springer Internat. Publishing (2014) ISBN 978-3-319-00211-8 Paul U. Nahin “Inside Interesting Integrals”, SpringerScience+Business Media (2015) ISBN 978-1-4939-1276-6, Chapt. 8, p. 279-341

[9] Dragoslav S. Mitrinović \& Jovan D. Kečkić “The Cauchy Method of Residues : Vol 1: Theory and its Applications" (Mathematics and its Applications), D. Reidel (1984) ISBN 90-277-1623-4

[10] Dragoslav S. Mitrinović \& Jovan D. Kečkić "The Cauchy Method of Residues : Vol 2: Theory and its Applications" (Mathematics and its Applications), Springer Science+Business Media (1993) ISBN-13: 978-94-010-4883-5 
Eric W. Weisstein MathWorld (2007) see: http://mathworld.wolfram.com/ResidueTheorem.html

[12] Wikipedia http://en.wikipedia.org/wicki/Residue theorem

[13] Michael Trott \& Oleg Marichev from The Wolfram Functions Site : http://functions.wolfram.com/

\section{About the Author}

Robert Kragler holds a doctoral degree in theoretical physics from University of Frankfurt/M. (1974) and a Dr. habil. from University of Konstanz (1981). He is professor from Weingarten University of Applied Sciences where he retired in 2008. He uses Mathematica since 1989 for his research and applied computer algebra methods particularly in his lectures courses on calculus and quantum physics .

\section{Robert Kragler}

Weingarten University of Applied Sciences

D-88241 Weingarten, Germany

kragler@hs-weingarten.de

http://portal.hs-weingarten.de/web/kragler/mathematica

Download : $\quad$ http://portal.hs-weingarten.de/web/kragler/Mathematica/ > Contour_Integration

Mma files (V10) : ContourIntegration_P1.nb, ContourIntegration.m 